\title{
Partial Bessel functions for quasi-split groups
}

\author{
J.W. Cogdell, I.I. Piatetski-Shapiro and F. Shahidi*
}

To Steve Rallis, with admiration, on the occasion of his sixtieth birthday

The question of functoriality is a central one in the theory of automorphic forms and representations. There has been recent progress on the proof of this conjecture for globally generic representations of the split classical groups by combining the converse theorem with the Langlands-Shahidi method of controlling automorphic $L$-functions [6, 7]. We refer the reader to those papers for an exposition of the general process.

One crucial local result needed in these proofs is the stability of the local $\gamma$-factors for the split classical groups. This seemingly technical result allows us to finesse the lack of the local Langlands conjecture at the finite number of finite places where the generic cuspidal representation is ramified. For the split odd orthogonal results this was established in [8] and then used in establishing functoriality from $\mathrm{SO}_{2 n+1}$ to $\mathrm{GL}_{2 n}$ in [6]. For the functoriality for even orthogonal groups and symplectic groups, the results of [8] were extended to these cases in Section 4 of [7]. In these later cases, the stability was established by expressing the local $\gamma$-factor as a Mellin transform of a certain partial Bessel function [16] and then using results on the asymptotics of these partial Bessel functions established in the split case in [8].

In order to facilitate the establishment of functoriality for general quasisplit groups, we turn to the problem of stability of local $\gamma$-factors for generic representations of these groups. In this paper, we extend the results of [8] and [7] on the asymptotics of certain partial Bessel functions of representations to the quasi-split case. The definition of the partial Bessel functions of interest can be found in Section 3 and the statement of our main result about them can be found in Section 7 of this paper. The arguments are essentially those found in [8] and [7] generalized to the quasi-split setting. Even though the modifications are minor in places, we have chosen to reproduce the arguments in full both to make this paper self contained and to be sure there is no question as to their validity in this increased generality.

\footnotetext{
* During the work on this project J.C. was partially supported by the NSA through grant MDA 904-03-1-0043 and by the Vaughn Foundation, I.I.P-S. by the NSF through grant DMS-0072450, and F.S. by the NSF through grant DMS-0200325.
} 
In a subsequent paper [9] we will turn to the combination of these asymptotics with the expression of the local $\gamma$-factor as a Mellin transform of related Bessel functions in [16] to obtain the general stability result for these $\gamma$-factors. Then progress on functoriality for quasi-split groups can proceed.

We conclude the paper with an Appendix on the existence of the (full) Bessel function of a generic representation associated to certain minimal Weyl elements (see Section 8). While this existence is not needed for the applications to functoriality, such Bessel functions are analogs of (twisted) orbital integrals (cf. Remark 3.2 of [16]) and are expected to play a fundamental role in the relative trace formula. Their existence was assumed in [16] (cf. Section 3 of that paper) for heuristic purposes, even though it was not used for the results established there; those results also require only the partial Bessel functions. We prove the existence of the Bessel function for quasi-split groups here, albeit in the limited context of certain minimal Weyl elements.

We would like to thank the referee for bringing to our attention a false proof in an earlier version of this paper and for the suggestions made on streamlining the presentation of the paper. We would also like to once again acknowledge that the genesis of many of the ideas for analyzing Bessel functions are to be found in the thesis of Averbuch [1].

\section{Preliminaries}

Let $k$ be a non-archimedean local field of characteristic zero, with ring of integers $\mathcal{O}$ and maximal ideal $\mathcal{P}$. Let $q=q_{k}$ be the order of the residue field $\mathcal{O} / \mathcal{P}$. Let $\Gamma=\operatorname{Gal}(\bar{k} / k)$ denote the absolute Galois group of $k$.

Let $\mathrm{G}$ be a connected reductive algebraic group which is defined and quasisplit over $k$. We shall assume in addition that the center $\mathrm{Z}=\mathcal{Z}(\mathrm{G})$ is connected and cohomologically trivial to first order, i.e., $H^{1}(\Gamma, Z)=\{0\}$. Since the ultimate goal of this paper is an application to the stability of the local $\gamma$-factor of a generic representation of $\mathrm{G}(k)$ under highly ramified twists, we know by Proposition 5.4 of [16] and the Appendix to [7] that these restrictions have no effect on the applicability of our results.

Fix a Borel subgroup $\mathrm{B}=\mathrm{TU}$ over $k$ with unipotent radical $\mathrm{U}$ and maximal torus $\mathrm{T}$. Let $\mathrm{A}$ be the maximal $k$-split subtorus of $\mathrm{T}$; then $\mathrm{T}=\mathcal{Z}(\mathrm{A})$.

Let $\widetilde{\Phi}=\Phi(\mathrm{T}, \mathrm{G})$ be the set of (non-restricted) roots of $\mathrm{T}$ in $\mathrm{G}[\mathbf{4}, \mathbf{1 3}]$. The choice of $\mathrm{U}$ then defines a set of positive roots $\widetilde{\Phi}^{+}$and simple roots $\widetilde{\Delta}$ of $\mathrm{T}$ in $\mathrm{U}$. Let $K / k$ be Galois splitting field of $\mathrm{G}$ and let $\Gamma_{K}=\operatorname{Gal}(K / k)$. Since $\mathrm{G}$ 
is quasi-split over $k$, and split over $K$, both $\widetilde{\Phi}$ and $\widetilde{\Delta}$ decompose into a finite number of $\Gamma$ or $\Gamma_{K}$ orbits [13].

Let $\Phi=\Phi(\mathrm{A}, \mathrm{G})$ be the set of (restricted) roots of $\mathrm{G}$ with respect to $\mathrm{A}$. Again, the choice of $\mathrm{U}$ then defines a set of positive roots $\Phi^{+}$and simple roots $\Delta$ for A. The root system $\Phi$ may have multiple roots. Let $\Phi_{n d}$ be the nondivisible roots of $\Phi$. For each $\alpha \in \Phi_{n d}$ we will let $(\alpha)$ denote the set of roots which are positive multiples of $\alpha$. The possibilities are either $(\alpha)=\{\alpha\}$ or $(\alpha)=\{\alpha, 2 \alpha\}[2]$. The $\Gamma$ or $\Gamma_{K}$ orbits in $\widetilde{\Delta}$ are in one-to-one correspondence with the restricted simple roots $\Delta$ [3]. Given $\alpha \in \Delta$ we will let $\widetilde{\Delta}_{\alpha}$ denote the $\Gamma$-orbit of roots $\tilde{\alpha} \in \widetilde{\Delta}$ which restrict to $\alpha$.

Let $W$ denote the (relative) Weyl group of $\mathrm{A}$ in $\mathrm{G}$, i.e., $\mathcal{N}(\mathrm{A}) / \mathcal{Z}(\mathrm{A})$.

1.1. Splittings. For each $\tilde{\alpha} \in \widetilde{\Delta}$ let $K_{\tilde{\alpha}}$ be the splitting field of the simple root $\tilde{\alpha}$. So $k \subset \mathrm{K}_{\tilde{\alpha}} \subset K$. Let $\left\{x_{\tilde{\alpha}} \mid \tilde{\alpha} \in \widetilde{\Delta}\right\}$ be a Steinberg splitting of $\mathrm{G}$ (cf. Section 4.1 .3 of [5]). So if $\mathrm{U}_{\tilde{\alpha}} \subset \mathrm{G}_{K}$ is the root subgroup corresponding to $\tilde{\alpha}$ over $K$, we have

(i) $x_{\tilde{\alpha}}: \mathbb{G}_{a} \rightarrow \mathrm{U}_{\tilde{\alpha}}$ is an isomorphism over $K_{\tilde{\alpha}}$;

(ii) $x_{\gamma(\tilde{\alpha})}=\gamma \circ x_{\tilde{\alpha}} \circ \gamma^{-1}$ for $\gamma \in \Gamma_{K}$.

This splitting determines an associated Chevalley system $\left\{x_{\tilde{\alpha}} \mid \tilde{\alpha} \in \widetilde{\Phi}\right\}$ for $\mathrm{G}$ over $K$ called a Chevalley-Steinberg system for $\mathrm{G}$ (see Section 4.1.3 of [5]). Recall that such a splitting always satisfies $A d(t) x_{\tilde{\alpha}}(u)=x_{\tilde{\alpha}}(\tilde{\alpha}(t) u)$ for $t \in \mathrm{T}(K)$. A Chevalley-Steinberg system then defines compatible root datum $\left(\mathrm{T},\left(\mathrm{U}_{\tilde{\alpha}}\right)_{\tilde{\alpha} \in \tilde{\Phi}}\right)$ for $\mathrm{G}(K)$ and $\left(\mathrm{T},\left(\mathrm{U}_{\alpha}\right)_{\alpha \in \Phi}\right)$ for $\mathrm{G}(k)$ [5]. The choice of a splitting gives representatives for the (absolute) Weyl group elements $w_{\tilde{\alpha}} \in \widetilde{W}=\mathcal{N}(T) / T$ associated to the simple reflections for $\tilde{\alpha} \in \widetilde{\Delta}$ via

$$
w_{\tilde{\alpha}}=x_{\tilde{\alpha}}(1) x_{-\tilde{\alpha}}(-1) x_{\tilde{\alpha}}(1)
$$

(see Section 3.2.1 of [5]).

The choice of a splitting fixes the natural homomorphisms from the usual simply connected rank one groups into G. If $\alpha \in \Delta$ is such that $(\alpha)=\{\alpha\}$ and we let $\tilde{\alpha} \in \widetilde{\Delta}$ be a root of $T$ restricting to $\alpha$, then the associated rank one group $\mathrm{G}^{\alpha}$ is isomorphic to $\mathrm{R}_{K_{\tilde{\alpha}} / k} S L_{2}$. In this case the associated $k$-splitting gives $x_{\alpha}=\mathrm{R}_{K_{\tilde{\alpha}} / k} x_{\tilde{\alpha}}$ and $x_{\alpha}: \mathrm{R}_{K_{\tilde{\alpha}} / k} \mathbb{G}_{a} \rightarrow \mathrm{U}_{\alpha}=\mathrm{U}_{(\alpha)}$. If $u \in K_{\tilde{\alpha}}$ then

$$
x_{\alpha}(u)=\prod_{\tilde{\beta} \in \tilde{\Delta}_{\alpha}} x_{\tilde{\beta}}\left(u_{\tilde{\beta}}\right) \quad \text { with } \quad u_{\gamma(\tilde{\alpha})}=\gamma(u) .
$$


If $\alpha \in \Delta$ is such that $(\alpha)=\{\alpha, 2 \alpha\}$ and we let $\tilde{\alpha}, \tilde{\alpha}^{\prime} \in \widetilde{\Delta}_{\alpha}$ be two roots of $\mathrm{T}$ restricting to $\alpha$ such that $\tilde{\alpha}+\tilde{\alpha}^{\prime}$ is again a root. Then $\tilde{\alpha}$ and $\tilde{\alpha}^{\prime}$ have the same splitting field, $K_{\tilde{\alpha}}$ which is a quadratic extension of the splitting field $K_{\tilde{\alpha}+\tilde{\alpha}^{\prime}}$ of $\tilde{\alpha}+\tilde{\alpha}^{\prime}$. For simplicity, let us denote $K_{\tilde{\alpha}}^{\prime}=K_{\tilde{\alpha}+\tilde{\alpha}^{\prime}}$. Then the associated rank one group $\mathrm{G}^{\alpha}$ is isomorphic to $\mathrm{R}_{K_{\tilde{\alpha}}^{\prime} / k} S U_{3}$. Let $\mathrm{H}_{\tilde{\alpha}}$ denote the subvariety of $K_{\tilde{\alpha}} \times K_{\tilde{\alpha}}$, considered as a vector space of dimension 4 over $K_{\tilde{\alpha}}^{\prime}$ defined by $v+v^{\sigma}=u^{\sigma} u$, where $\sigma$ is the non-trivial Galois automorphism in $\operatorname{Gal}\left(K_{\tilde{\alpha}} / K_{\tilde{\alpha}}^{\prime}\right)$, equipped with the group law

$$
(u, v)\left(u^{\prime}, v^{\prime}\right)=\left(u+u^{\prime}, v+v^{\prime}+u^{\sigma} u^{\prime}\right) .
$$

Then $\mathrm{U}_{(\alpha)} \simeq \mathrm{R}_{K_{\tilde{\alpha}} / K_{\tilde{\alpha}}^{\prime}} \mathrm{H}_{\tilde{\alpha}}$, with the pair $(u, v)$ corresponding to the unipotent matrix

$$
\mu(u, v)=\left(\begin{array}{ccc}
1 & -u^{\sigma} & -v \\
0 & 1 & u \\
0 & 0 & 1
\end{array}\right) \in S U_{3} .
$$

Then the splitting is given as follows. Choose a splitting $x_{\tilde{\alpha}}^{\prime}: \mathbb{G}_{a} \rightarrow \mathrm{U}_{\tilde{\alpha}}$. Then $x_{\tilde{\alpha}^{\prime}}=\sigma \circ x_{\tilde{\alpha}} \circ \sigma^{-1}$ and $x_{\tilde{\alpha}+\tilde{\alpha}^{\prime}}=\operatorname{int} w_{\tilde{\alpha}^{\prime}}^{-1} \circ x_{\tilde{\alpha}}$ (see [5], Sction 4.1.9). Then

$$
x_{\alpha}(u, v)=\prod_{\left\{\tilde{\beta}, \tilde{\beta}^{\prime}\right\}} x_{\tilde{\beta}}\left(u_{\tilde{\beta}}\right) x_{\tilde{\beta}+\tilde{\beta}^{\prime}}\left(-v_{\tilde{\beta}}\right) x_{\tilde{\beta}^{\prime}}\left(u_{\tilde{\beta}}^{\sigma}\right)
$$

for $(u, v) \in \mathrm{H}_{\tilde{\alpha}} \subset K_{\tilde{\alpha}} \times K_{\tilde{\alpha}}$, where the product is over distinct pairs $\left\{\tilde{\beta}, \tilde{\beta}^{\prime}\right\} \in$ $\widetilde{\Delta}_{\alpha}$ with $\tilde{\beta}+\tilde{\beta}^{\prime}$ a root. Here, for each $\tilde{\beta}$ we choose $\gamma \in \operatorname{Gal}(K / k)$ such that $\tilde{\beta}=\gamma(\tilde{\alpha})$; then $\tilde{\beta}^{\prime}=\gamma\left(\tilde{\alpha}^{\prime}\right), x_{\tilde{\beta}}=\gamma \circ x_{\tilde{\alpha}} \circ \gamma^{-1}, x_{\tilde{\beta}^{\prime}}=\gamma \circ x_{\tilde{\alpha}^{\prime}} \circ \gamma^{-1}$, $x_{\tilde{\beta}+\tilde{\beta}^{\prime}}=\gamma \circ x_{\tilde{\alpha}+\tilde{\alpha}^{\prime}} \circ \gamma^{-1}, u_{\tilde{\beta}}=\gamma(u)$, and $v_{\tilde{\beta}}=\gamma(v)$. Note that the image of $x_{\tilde{\alpha}}(u, v)$ in $\mathrm{U}_{\alpha} / \mathrm{U}_{2 \alpha}$ only depends on $u$ and will be denoted $\bar{x}_{\alpha}(u)$. The map $u \mapsto \bar{x}_{\alpha}(u)$ gives an isomorphism of $k$-vector spaces of $K_{\tilde{\alpha}}$ onto $\mathrm{U}_{(\alpha)} / \mathrm{U}_{2 \alpha}$. We shall use this notation in the case of $(\alpha)=\{\alpha\}$ as well, taking $\mathrm{U}_{2 \alpha}$ to be trivial.

The splitting, through the isomorphisms with the simply connected rank one groups, gives representatives for the (relative) Weyl group elements $w_{\alpha} \in$ $W$ associated to the simple reflections for $\alpha \in \Delta$ (see Sections 4.1.5 and 4.1.9 of [5]). We can then choose representatives for each $w \in W$ by means of a reduced decomposition and this choice of the $w_{\alpha}$. This is independent of the choice of decomposition.

Let $\omega: k^{\times} \rightarrow \mathbb{Z}$ be the valuation on $k$, with associated normalized absolute value $|u|=q_{k}^{-\omega(u)}$ where $q_{k}$ is the order of the residue class field of $k$. This extends uniquely to give a compatible valuation on $K$ and each $K_{\tilde{\alpha}}$, which we will also denote by $\omega$ since it is unique, with associated absolute values. The root datum $\left(\mathrm{T},\left(\mathrm{U}_{\tilde{\alpha}}\right)_{\tilde{\alpha} \in \tilde{\Phi}}\right)$ is naturally valued by the maps $\varphi_{\tilde{\alpha}}: \mathrm{U}_{\tilde{\alpha}} \rightarrow \mathbb{R}$ 
defined by

$$
\varphi_{\tilde{\alpha}}\left(x_{\tilde{\alpha}}(u)\right)=\omega(u) \quad \text { for } u \in K^{\times} .
$$

This valuation on the root datum descends to a valuation $\varphi_{\alpha}: \mathrm{U}_{\alpha} \rightarrow \mathbb{R}$ of the associated root datum $\left(\mathrm{T},\left(\mathrm{U}_{\alpha}\right)_{\alpha \in \Phi}\right)$ for $\mathrm{G}(k)$ (see Section 4.2.2 and Theorem 4.2.3 of [5]). If $\alpha \in \Delta$ is such that $(\alpha)=\{\alpha\}$ then we have

$$
\varphi_{\alpha}\left(x_{\alpha}(u)\right)=\omega(u) \quad \text { for } u \in K_{\tilde{\alpha}}^{\times} .
$$

If the root $\alpha \in \Delta$ is multiple, so $(\alpha)=\{\alpha, 2 \alpha\}$, then

$$
\varphi_{\alpha}\left(x_{\alpha}(u, v)\right)=\frac{1}{2} \omega(v) \leq \omega(u) \quad \text { for } \quad(u, v) \in \mathrm{H}_{\tilde{\alpha}}, \quad(u, v) \neq(0,0)
$$

while

$$
\varphi_{2 \alpha}\left(x_{\alpha}(0, v)\right)=\omega(v) \quad \text { for } \quad v \in K_{\tilde{\alpha}}^{\times}, \operatorname{Tr}_{K_{\tilde{\alpha}} / K_{\tilde{\alpha}}^{\prime}}(v)=0
$$

The valued root datum allows us to define a natural exhaustive family of compact open subgroups of $\mathrm{U}(K)$ and hence $\mathrm{U}(k)$. Enumerate the simple roots $\widetilde{\Delta}=\left\{\tilde{\alpha}_{1}, \ldots, \tilde{\alpha}_{r}\right\}$. If $\tilde{\beta} \in \widetilde{\Phi}^{+}$is a positive root occurring in $\mathrm{U}$ then $\tilde{\beta}$ has a unique expression of the form $\tilde{\beta}=n_{1} \tilde{\alpha}_{1}+\cdots+n_{r} \tilde{\alpha}_{r}$ with $n_{j}$ a nonnegative integer. Then as usual we set $\operatorname{ht}(\tilde{\beta})=n_{1}+\cdots+n_{r}$, the height of the positive root $\tilde{\beta}$. For each positive integer $M$ we define a concave function $f_{M}: \widetilde{\Phi} \rightarrow \mathbb{R} \cup\{\infty\}$ by $f_{M}(\tilde{\beta})=-\operatorname{ht}(\tilde{\beta}) M$ if $\tilde{\beta} \in \widetilde{\Phi}^{+}$and $f_{M}(\tilde{\beta})=\infty$ if $\tilde{\beta} \in \widetilde{\Phi}^{-}$. This then allows us to define an open compact subgroup $\mathrm{U}_{\tilde{\beta}, f_{M}} \subset$ $\mathrm{U}_{\tilde{\alpha}}(K)$ by $\mathrm{U}_{\tilde{\beta}, f_{M}}=\varphi_{\tilde{\beta}}^{-1}\left(\left[f_{M}(\tilde{\beta}), \infty\right]\right)$ (see Section 6.2 of $\left.[4]\right)$. If $\tilde{\beta} \in \widetilde{\Phi}^{+}$ then $\mathrm{U}_{\tilde{\beta}, f_{M}}=\varphi_{\tilde{\beta}}^{-1}([M \operatorname{ht}(\tilde{\beta}), \infty])$ while if $\tilde{\beta} \in \widetilde{\Phi}^{-}$then $\mathrm{U}_{\tilde{\beta}, f_{M}}=\{1\}$. Then, following Bruhat and Tits, we define a corresponding subgroup $\mathrm{U}_{f_{M}} \subset \mathrm{G}(K)$ as the subgroup generated the $\mathrm{U}_{\tilde{\beta}, f_{M}}$ (see Section 6.4 of [4]). In our case, this will be a compact open subgroup of $\mathrm{U}(K)$ and as $M \rightarrow \infty$ these will exhaust $\mathrm{U}(K)$. As the standard commutation relations show (see also Section 6.1 of [4]) in our case we can describe $\mathrm{U}_{f_{M}}$ simply as those elements of $\mathrm{U}(K)$ of the form

$$
u=\prod_{\tilde{\beta} \in \tilde{\Phi}^{+}} x_{\tilde{\beta}}\left(u_{\tilde{\beta}}\right) \quad \text { with }\left|u_{\tilde{\beta}}\right|_{K} \leq q_{K}^{M \operatorname{ht}(\tilde{\beta})} .
$$

Since the valued root datum descends to $\left(\mathrm{T},\left(\mathrm{U}_{\beta}\right)_{\beta \in \Phi}\right)$ and the function $f_{M}$ is Galois invariant, the subgroups $\mathrm{U}_{f_{M}}$ will also descend to subgroups $\mathrm{U}_{f_{M}} \subset$ $\mathrm{U}(k)$. These will play a role in what follows. This family of open compact subgroups will also satisfy the conditions needed in [16] (see particularly Section 6 therein). 
1.2. Generic characters of $\mathbf{U}$. The notion of splitting is also necessary to define the concept of a generic character of $\mathrm{U}(k)$ and generic representations. Let $\psi$ be a non-trivial additive character of $k$. If $u \in \mathrm{U}(k)$ then we can write

$$
u=\prod_{\tilde{\beta} \in \widetilde{\Phi}^{+}} x_{\tilde{\beta}}\left(u_{\tilde{\beta}}\right)
$$

with the $u_{\tilde{\beta}} \in K$ satisfying $\gamma\left(u_{\tilde{\beta}}\right)=u_{\gamma(\tilde{\beta})}$ for all $\gamma \in \Gamma_{K}$. Then we can extend $\psi$ to a non-degenerate character of $\mathrm{U}(k)$ relative to this splitting by setting

$$
\psi(u)=\psi\left(\sum_{\tilde{\alpha} \in \widetilde{\Delta}} u_{\tilde{\alpha}}\right) .
$$

Note that the Galois invariance of the $\widetilde{\Delta}$ ensures that $\sum u_{\tilde{\alpha}} \in k$.

The abelianization $\mathrm{U}^{a b}$ of $\mathrm{U}$ is isomorphic to the direct sum of the abelianization of the root groups $\mathrm{U}_{(\alpha)}$ for $\alpha \in \Delta$ and we have $\mathrm{U}_{(\alpha)}^{a b} \simeq \mathrm{U}_{(\alpha)} / \mathrm{U}_{2 \alpha} \simeq$ $\mathrm{R}_{K_{\tilde{\alpha} / k}} \mathbb{G}_{a}$, with the last isomorphism being given by $u_{\tilde{\alpha}} \in K_{\tilde{\alpha}} \mapsto \bar{x}_{\alpha}\left(u_{\tilde{\alpha}}\right)$. Thus we have

$$
\psi(u)=\psi\left(\sum_{\alpha \in \Delta} \bar{x}_{\alpha}\left(u_{\tilde{\alpha}}\right)\right)=\prod_{\alpha \in \Delta} \psi\left(\operatorname{Tr}_{K_{\tilde{\alpha}} / k}\left(u_{\tilde{\alpha}}\right)\right)
$$

If we let $\psi_{\alpha}=\psi \circ \operatorname{Tr}_{K_{\tilde{\alpha}} / k}$ then we see that under the isomorphism $\mathrm{U}^{a b} \simeq$ $\oplus \mathrm{R}_{K_{\tilde{\alpha}} / k} \mathbb{G}_{a}$ we have $\psi=\prod \psi_{\alpha}$.

The representatives of $w \in W$ fixed above will be compatible with $\psi$ in the following sense. For every subset $\theta \subset \Delta$ and $w \in W$ such that $w(\theta) \subset \Delta$ we have

$$
\psi(u)=\psi(A d(w) u)
$$

for all $u \in \mathrm{U}_{\theta}$, the unipotent radical of the Levi subgroup $\mathrm{M}_{\theta}$ of the parabolic subgroup $\mathrm{P}_{\theta}$ associated to $\theta$ (see Section 1.4 below and Section 3 of [15]). When we speak of generic representations of $\mathrm{G}(k)$ we will always mean generic with respect to this character $\psi$ of $\mathrm{U}(k)$.

1.3. A splitting of the torus. Recall that we have assumed that the center $\mathrm{Z}=\mathcal{Z}(\mathrm{G})$ of $\mathrm{G}$ is connected and cohomologically trivial to first order, i.e., $H^{1}(\Gamma, \mathrm{Z})=\{0\}$.

Enumerate the (non-restricted) simple roots of $\mathrm{T}$ as $\widetilde{\Delta}=\left\{\tilde{\alpha}_{1}, \ldots, \tilde{\alpha}_{r}\right\}$. Let $K_{\tilde{\alpha}_{i}}=K_{i} \supset \mathcal{O}_{i} \supset \mathcal{P}_{i}$ denote the ring of integers and maximal ideal for the field of definition of $\tilde{\alpha}_{i}$. Then for every $t \in \mathrm{T}(k)$ we have $\tilde{\alpha}_{i}(t) \subset K_{i}$. For 
every $n$-tuple $\underline{M}=\left(M_{1}, \ldots, M_{r}\right)$ of positive integers let us set

$$
\mathrm{T}_{\underline{M}}=\left\{t \in \mathrm{T}(k) \mid \tilde{\alpha}_{i}(t) \in 1+\mathcal{P}_{i}^{M_{i}}, 1 \leq i \leq r\right\} .
$$

Note that $\mathrm{Z}(k) \subset \mathrm{T}_{M}$ for all $\underline{M}$. For later purposes (see Section 6) we would like to be able to split the center off of $\mathrm{T}_{\underline{M}}$ for $\underline{M}$ sufficiently large.

We have the short exact sequence

$$
0 \longrightarrow \mathrm{Z} \longrightarrow \mathrm{T} \stackrel{\rho}{\longrightarrow} \mathrm{T}_{a d} \longrightarrow 0
$$

where $\mathrm{T}_{a d}$ is a Cartan in the adjoint group of $\mathrm{G}$. We can take $\rho$ to be

$$
\rho(t)=\left(\tilde{\alpha}_{1}(t), \ldots, \tilde{\alpha}_{r}(t)\right) \in(\bar{k})^{r} .
$$

Then

$$
\mathrm{T}_{a d} \simeq\left\{\left(\tilde{\alpha}_{1}(t), \ldots, \tilde{\alpha}_{r}(t)\right) \mid t \in \mathrm{T}\right\} .
$$

Any exact sequence of tori splits. Let $j$ be a splitting, i.e., an injection $j$ : $\mathrm{T}_{a d} \rightarrow \mathrm{T}$ such that $\rho \circ j=i d$. Applying cohomology we find

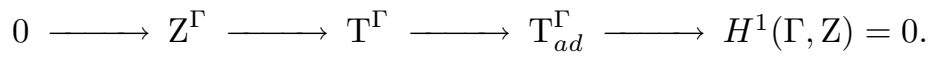

Thus

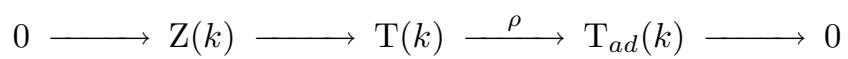

is exact and split and consequently

$$
\mathrm{T}(k) \simeq \mathrm{Z}(k) \mathrm{T}_{a d}(k) .
$$

Observe that $\mathrm{Z}(k)$ is the center of $\mathrm{G}(k)$. Moreover

$$
\mathrm{T}_{a d}(k) \simeq\left\{\left(\tilde{\alpha}_{1}(t), \ldots, \tilde{\alpha}_{r}(t)\right) \mid t \in \mathrm{T}(k)\right\} .
$$

Thus

$$
\mathrm{T}_{a d}(k) \subset K_{1}^{\times} \times \cdots \times K_{r}^{\times}
$$

where $K_{i} / k$ are as above. Let

$$
O_{i}=\left\{\tilde{\alpha}_{i}(t) \mid t \in \mathrm{T}(k)\right\} \subset K_{i}^{\times} .
$$

Observe that each $O_{i}$ is open in $K_{i}^{\times}$. Take the $M_{i}$ large enough that

$$
\left(1+\mathcal{P}_{1}^{M_{1}}\right) \times \cdots \times\left(1+\mathcal{P}_{r}^{M_{r}}\right) \subset \mathrm{T}_{a d}(k)
$$

and let

$$
\mathrm{T}_{\underline{M}}^{1}=j\left(\left(1+\mathcal{P}_{1}^{M_{1}}\right) \times \cdots \times\left(1+\mathcal{P}_{r}^{M_{r}}\right)\right) .
$$

Observe that

$$
\rho\left(\mathrm{T}_{\underline{M}}\right)=\left(1+\mathcal{P}_{1}^{M_{1}}\right) \times \cdots \times\left(1+\mathcal{P}_{r}^{M_{r}}\right)
$$


and then

$$
T_{\underline{M}}=Z(k) \mathrm{T}_{\underline{M}}^{1} .
$$

Thus we have proved the following lemma.

Lemma 1.1. With the notation above, for $\underline{M}=\left(M_{1}, \ldots, M_{n}\right)$ sufficiently large we have the splitting

$$
T_{\underline{M}}=Z(k) \mathrm{T}_{\underline{M}}^{1} .
$$

We finally note that if $\mathrm{G}$ is as above and its center $\mathrm{Z}$ has $H^{1}(\Gamma, \mathrm{Z})=\{0\}$, then the same will be true for the center $\mathrm{Z}(\mathrm{M})$ of any Levi subgroup $\mathrm{M}$ of $\mathrm{G}$. This follows by using induced tori to which one can apply Shapiro's lemma. Thus we have similar splittings for the maximal tori of any Levi subgroup of G. We will present a detailed proof of this in [9].

1.4. Bruhat decomposition. Let $W$ denote the (relative) Weyl group of A in G, i.e., $\mathcal{N}(\mathrm{A}) / \mathcal{Z}(\mathrm{A})$ and let $S$ denote the set of simple reflections in $W$ corresponding to the choice of simple roots $\Delta$ as above. Then $(\mathrm{G}(k), \mathrm{B}(k), \mathrm{N}(k), S)$ is a Tits system, where we have let $\mathrm{N}=\mathcal{N}(\mathrm{A})$, and the pair $(W, S)$ is a Coxeter system [2]. Hence the basic results on the Bruhat decomposition remain valid in this case.

We recall some standard results, all of which can be found in Section 21 of [2]. Let $\mathfrak{g}$ be the Lie algebra of G. For $\alpha \in \Phi$, let $\mathfrak{g}_{\alpha}$ be the corresponding eigenspace in $\mathfrak{g}$ and $\mathfrak{g}_{(\alpha)}=\oplus_{\beta \in(\alpha)} \mathfrak{g}_{\beta}$. For each $\alpha$ there is a unique closed unipotent $k$-subgroup $\mathrm{U}_{(\alpha)}$ normalized by $\mathrm{T}$ with Lie algebra $\mathfrak{g}_{(\alpha)}$. (These were described in Section 1.1 for $\alpha \in \Delta$.) $\mathrm{U}$ is then directly spanned by the $\mathrm{U}_{(\alpha)}$ with $\alpha \in \Phi_{n d}^{+}$.

For $w \in W$ let

$$
\Phi_{w}^{+}=\left\{\alpha \in \Phi_{n d}^{+} \mid w(\alpha)>0\right\} \quad \text { and } \quad \Phi_{w}^{-}=\left\{\alpha \in \Phi_{n d}^{+} \mid w(\alpha)<0\right\}
$$

and set

$$
\mathrm{U}_{w}^{+}=\mathrm{U}_{\Phi_{w}^{+}}=\mathrm{U} \cap w^{-1} \mathrm{U} w \quad \text { and } \quad \mathrm{U}_{w}^{-}=\mathrm{U}_{\Phi_{w}^{-}}=\mathrm{U} \cap w^{-1} \mathrm{U}^{-} w .
$$

Then

$$
\mathrm{U}=\mathrm{U}_{w}^{+} \mathrm{U}_{w}^{-}
$$

and

$$
w \mathrm{U}_{w}^{+} w^{-1} \subset \mathrm{U} \text { and } \quad w \mathrm{U}_{w}^{-} w^{-1} \subset \mathrm{U}^{-} .
$$


For each $w \in W$ we let $C(w)=\mathrm{B} w \mathrm{~B}=\mathrm{B} w \mathrm{U}_{w}^{-}$be the corresponding Bruhat cell. The ( $k$-rational) Bruhat decomposition for $G$ is then

$$
G=\bigcup_{w \in W} C(w)=\bigcup_{w \in W} \mathrm{~B} w \mathrm{U}_{w}^{-} .
$$

The relative closure of the Bruhat cells are described as follows (see Theorem 21.26 and Proposition 21.27 of [2]). For $w \in W$, let $w=w_{\alpha_{1}} \cdots w_{\alpha_{n}}, \alpha_{i} \in \Delta$, be a reduced decomposition of $w$. Let

$$
S(w)=\left\{w_{\alpha_{i_{1}}} \cdots w_{\alpha_{i_{m}}} \mid 1 \leq i_{1}<\cdots<i_{m} \leq n\right\} .
$$

Then

$$
\overline{C(w)}=\bigcup_{w^{\prime} \in S(w)} C\left(w^{\prime}\right) .
$$

Since the Bruhat order on $W$ can be characterized by $w^{\prime} \leq w$ iff $w^{\prime} \in S(w)$ [12], we may also write this as

$$
\overline{C(w)}=\bigcup_{w^{\prime} \leq w} C\left(w^{\prime}\right) .
$$

Let $\theta \subset \Delta$. Let $[\theta]$ denote the set of $k$-roots which are linear combinations of the elements of $\theta$. Set $\Psi(\theta)=\Phi^{+}-[\theta]$. Let

$$
\mathrm{A}_{\theta}=\left(\bigcap_{\alpha \in[\theta]} \operatorname{ker} \alpha\right)^{\circ} \text {. }
$$

Then $[\theta]=\Phi\left(\mathrm{A}, \mathcal{Z}\left(\mathrm{A}_{\theta}\right)\right)$ and $\mathcal{Z}\left(\mathrm{A}_{\theta}\right)=\left\langle\mathrm{T}, \mathrm{U}_{(\alpha)} \mid \alpha \in[\theta]\right\rangle$. Then

$$
\mathrm{P}_{\theta}=\mathcal{Z}\left(\mathrm{A}_{\theta}\right) \ltimes \mathrm{U}_{\Psi(\theta)}
$$

is a standard parabolic subgroup. $\mathrm{P}_{\emptyset}=\mathrm{B}$. If we set $W_{\theta}=\left\langle w_{\alpha} \mid \alpha \in \theta\right\rangle$ then we also have

$$
\mathrm{P}_{\theta}=\mathrm{B} W_{\theta} \mathrm{B} .
$$

If $\alpha$ is a simple root and we let $\mathrm{P}_{\alpha}=\mathrm{P}_{\{\alpha\}}$ then

$$
\mathrm{P}_{\alpha}=C\left(w_{\alpha}\right) \cup \mathrm{B} .
$$

\section{Partial Bessel functions}

2.1. Finite field heuristics. In order to motivate what follows, let us take $\mathbb{F}$ to be a finite field and $G$ a quasi-split reductive algebraic group over $\mathbb{F}$. The 
basics of the theory of Bessel functions for representations of algebraic groups over finite fields can be found in $[\mathbf{1 0}, \mathbf{1 1}]$.

We may retain all the notation and concepts of Section 1. Suppose that $\left(\pi, V_{\pi}\right)$ is a generic representation of $\mathrm{G}(\mathbb{F})$. Over a finite field, generic representations will tend to have both Whittaker functionals and Whittaker vectors. So suppose that $v_{W} \in V_{\pi}$ satisfies $\pi(u) v_{W}=\psi(u) v_{W}$ for $u \in \mathrm{U}(\mathbb{F})$ and $\Lambda=\Lambda_{W}$ satisfies $\Lambda(\pi(u) v)=\psi(u) \Lambda(v)$ for all $u \in \mathrm{U}(\mathbb{F})$ and all $v \in V_{\pi}$. Moreover, assume these choices are normalized so that $\Lambda\left(v_{W}\right)=1$. Then $J_{\pi}(g)=\Lambda\left(\pi(g) v_{W}\right)$ is a function in the Whittaker model of $\pi$ and it satisfies

$$
J_{\pi}\left(u g u^{\prime}\right)=\psi(u) J_{\pi}(g) \psi\left(u^{\prime}\right) \quad u, u^{\prime} \in \mathrm{U}(\mathbb{F}) .
$$

This is the Bessel function of the representation.

If we restrict this function to the various Bruhat cells, then the restriction of $J_{\pi}$ to $C(w)$ is not identically zero iff for every $\alpha \in \Delta$ we have that $w \alpha>0$ implies $w \alpha \in \Delta$. Let $J_{\pi, w}$ denote the restriction of $J_{\pi}$ to $C(w)$. Write $C(w)=$ $\mathrm{B} w \mathrm{~B}=\mathrm{UT} w \mathrm{U}_{w}^{-}$. Then $J_{\pi, w}\left(u t w u^{\prime}\right)=\psi(u) J_{\pi, w}(t w) \psi\left(u^{\prime}\right)$ is essentially a function on $\mathrm{T}(\mathbb{F})$ and one can check that its restriction to the split $\mathrm{A}$ vanishes unless $a \in \mathrm{A}_{w}=\{a \in \mathrm{A} \mid w \alpha(a)=1$ for all simple $\alpha$ with $w \alpha>0\}$. This function on $A_{w}$ carries the information of the restriction $J_{\pi, w}$ of the Bessel function $J_{\pi}$ to the Bruhat cell associated to $w$. by

Finally, for what follows, note that one has a formula for $J_{\pi, w}(a w)$ given

$$
J_{\pi, w}(a w)=\left|\mathrm{U}_{w}^{-}(\mathbb{F})\right|^{-1} \int_{\mathrm{U}_{w}^{-}(\mathbb{F})} W_{v_{W}}(a w u) \psi^{-1}(u) d u
$$

where, as usual, the Whittaker model is defined by $W_{v}(g)=\Lambda(\pi(g) v)$ for $v \in V_{\pi}$.

2.2. Weyl group elements that support Bessel functions. Now let $k$ once again be a non-archimedean field of characteristic 0 .

We say that an element $w \in W$ supports a Bessel function if for every $\alpha \in \Delta$ we have that $w \alpha>0$ implies $w \alpha \in \Delta$. So every simple root which remains positive under the action of $w$ must remain simple. Note that $w=e$ and $w=w_{\ell}$, the long element of $W$, always support Bessel functions. By Lemma 89 of [17] (page 257), which is valid for quasi-split groups, we have that $w$ supports a Bessel function iff $w=w_{\ell} w_{\ell}^{\theta}$ for $\theta=\theta_{w}=\{\alpha \in \Delta \mid w \alpha>0\} \subset \Delta$ and $w_{\ell}^{\theta} \in W_{\theta}$ the corresponding long Weyl element. This implies that there are actually $2^{|\Delta|}$ Weyl elements which support Bessel functions and to each $\theta \subset \Delta$ we have associated a Weyl element $w_{\theta}=w_{\ell} w_{\ell}^{\theta}$ which supports a Bessel function. 
To the set $\theta$ is associated the standard parabolic subgroup $\mathrm{P}_{\theta}$ as above. Then, in this case, we have $\Phi_{w}^{+}=\left[\theta_{w}\right]$ so that $\mathrm{U}_{w}^{+}=\mathrm{U}_{\theta}$ and $\mathrm{U}_{w}^{-}=\mathrm{U}_{\Psi(\theta)}$. Furthermore, if we set

$$
\begin{aligned}
\mathrm{A}_{w} & =\{a \in \mathrm{A} \mid w \alpha(a)=1 \text { for all simple } \alpha \text { with } w \alpha>0\} \\
& =\{a \in \mathrm{A} \mid w \alpha(a)=1 \text { for all } \alpha \in \theta\}
\end{aligned}
$$

then $\mathrm{A}_{w}^{0}=\mathrm{A}_{w(\theta)}$ is the split component of the center of the Levi component of the associate parabolic $\mathrm{P}_{w(\theta)}$. (Note that in [8] we implicitly assumed that $w(\theta)=\theta$, which was indeed true in the case of interest to us.)

We recall from [8] the following proposition.

Proposition 2.1. Let $w, w^{\prime} \in W$ support Bessel functions. Write $w=w_{\ell} w_{\ell}^{\theta}$ and $w^{\prime}=w_{\ell} w_{\ell}^{\theta^{\prime}}$ for subsets $\theta, \theta^{\prime} \subset \Delta$. Then $w^{\prime} \leq w$ iff $\theta \subset \theta^{\prime}$. In particular, if $\theta$ is of the form $\theta=\Delta-\{\alpha\}$, i.e., if $\mathrm{P}_{\theta}$ is a maximal parabolic subgroup, then the only Weyl elements $w^{\prime} \leq w$ which support Bessel functions are $w^{\prime}=w$ and $w^{\prime}=e$.

Proof: Let $\theta=\theta_{w}$ and $\theta^{\prime}=\theta_{w^{\prime}}$. By Example 3, Section 5.9 of [12], we know that $w^{\prime} \leq w$ iff $w_{\ell} w \leq w_{\ell} w^{\prime}$ and this last is equivalent to $w_{\ell}^{\theta} \leq w_{\ell}^{\theta^{\prime}}$. Since $w_{\ell}^{\theta}$ is the long element of $W_{\theta}$, then by the compatibility of the Bruhat ordering (Section 5.5 and 5.10 of [12]) any reduced expression for $w_{\ell}^{\theta}$ contains only the basic reflections $r_{\alpha}$ for $\alpha \in \theta$ and by Section 1.8 of [12], we know that each simple reflection $r_{\alpha}$ with $\alpha \in \theta$ occurs. The same is true of $w_{\ell}^{\theta^{\prime}}$ with respect to $\theta^{\prime}$. However, $w_{\ell}^{\theta} \leq w_{\ell}^{\theta^{\prime}}$ can be characterized by $w_{\ell}^{\theta}$ occurring as a sub-expression of a reduced expression for $w_{\ell}^{\theta^{\prime}}$. Thus we must have $\theta \subset \theta^{\prime}$.

We will say that $w \in W$ is a minimal Weyl element supporting a Bessel function if $w$ supports a Bessel function and the only $w^{\prime} \in W$ with $w^{\prime} \leq w$ which support Bessel functions are $w^{\prime}=w$ and $w^{\prime}=e$, i.e., the associated parabolic subgroup is maximal.

2.3. Bessel functions. Let $\left(\pi, V_{\pi}\right)$ be an irreducible admissible generic representation of $\mathrm{G}(k)$. We fix a splitting of $\mathrm{U}$ and a non-degenerate character $\psi$ of $\mathrm{U}(k)$ as in Section 1.2. Let $\mathcal{W}(\pi, \psi)$ be the associated Whittaker model of $\pi$. The functions $W \in \mathcal{W}(\pi, \psi)$ satisfy $W(u g)=\psi(u) W(g)$ for $u \in \mathrm{U}(k)$.

If $\pi$ is a generic representation with Whittaker model $\mathcal{W}(\pi, \psi)$ then to each $w \in W$ which supports a Bessel function we may associate a formal Bessel function $J_{\pi, w}(a)$ for $a \in A_{w}$ by

$$
J_{\pi, w}(a)=\int_{\mathrm{U}_{w}^{-}(k)} W_{v}(a w u) \psi^{-1}(u) d u
$$


for any choice of $W_{v} \in \mathcal{W}(\pi, \psi)$ for which $W_{v}(e)=1$.

Assuming that the integral exists, this is independent of the choice of $v \in$ $V_{\pi}$, since the map

$$
v \mapsto \int_{\mathrm{U}_{w}^{-}(k)} W_{v}(a w u) \psi^{-1}(u) d u
$$

defines a $\psi$-Whittaker functional on $V_{\pi}$. To see this, consider $\pi\left(u^{\prime}\right) v$. Under the decomposition $\mathrm{U}=\mathrm{U}_{w}^{+} \mathrm{U}_{w}^{-}$we write $u^{\prime}=u^{\prime}{ }^{\prime} u^{\prime}$. Then

$$
\begin{aligned}
\int_{\mathrm{U}_{w}^{-}(k)} W_{\pi\left(u^{\prime}\right) v}(a w u) & \psi^{-1}(u) d u=\int_{\mathrm{U}_{w}^{-}(k)} W_{v}\left(a w u u^{\prime}\right) \psi^{-1}(u) d u \\
& =\int_{\mathrm{U}_{w}^{-}(k)} W_{v}\left(a w u^{\prime}+u u^{\prime}\right) \psi^{-1}(u) d u \\
& =\psi\left(u^{\prime}\right) \int_{\mathrm{U}_{w}^{-}(k)} W_{v}\left(a w u^{\prime+} u\right) \psi^{-1}(u) d u \\
& =\psi\left(u^{\prime}\right) \psi\left(A d(a w) u^{\prime}\right) \int_{\mathrm{U}_{w}^{-}(k)} W_{v}(a w u) \psi^{-1}(u) d u \\
& =\psi\left(u^{\prime}\right) \psi\left(A d(a w) u^{\prime}\right) \int_{\mathrm{U}_{w}^{-}(k)} W_{v}(a w u) \psi^{-1}(u) d u .
\end{aligned}
$$

Now, since $u^{\prime} \in \mathrm{U}_{w}^{+}=\mathrm{U}_{\theta}$, then $A d(w) u^{\prime+} \in \mathrm{U}_{w(\theta)}$. Also, $a \in A_{w}=$ $\mathcal{Z}\left(M_{w(\theta)}\right)$. Thus $A d(a w) u^{\prime+}=A d(w) u^{\prime}+$ and so by compatibility

$$
\psi\left(\operatorname{Ad}(a w) u^{\prime+}\right)=\psi\left(\operatorname{Ad}(w) u^{\prime}\right)=\psi\left(u^{\prime}\right) .
$$

Hence

$$
\int_{\mathrm{U}_{w}^{-}(k)} W_{\pi\left(u^{\prime}\right) v}(a w u) \psi^{-1}(u) d u=\psi\left(u^{\prime}\right) \int_{\mathrm{U}_{w}^{-}(k)} W_{v}(a w u) \psi^{-1}(u) d u
$$

and this integral defines a Whittaker functional on $V_{\pi}$. By the uniqueness of the Whittaker model, this must be a non-zero multiple of the standard Whittaker functional $v \mapsto W_{v}(e)$. So there is a constant $J_{\pi, w}(a)$

$$
\int_{\mathrm{U}_{w}^{-}(k)} W_{v}(a w u) \psi^{-1}(u) d u=J_{\pi, w}(a) W_{v}(e) .
$$

Since $v$ was chosen so that $W_{v}(e)=1$ this gives

$$
\int_{\mathrm{U}_{w}^{-}(k)} W_{v}(a w u) \psi^{-1}(u) d u=J_{\pi, w}(a)
$$

independent of $v$ with this property. 
The convergence of the integrals defining the Bessel functions is subtle and in general we do not have a proof. For Bessel functions attached to a minimal Weyl element which supports a Bessel function we gave an argument for convergence in [8] for the case of split groups (see Lemmas 4.2 and 4.3 as well as Proposition 4.2 of [8]). The convergence for the quasi-split case can be proven by the same argument, utilizing the compact open subgroups $\mathrm{U}_{f_{M}}$ of Section 1.1 in place of the corresponding $X(M)$ of [8]. Since we will not use this full Bessel function in the main theorem or its applications, we will forgo presenting the details of the proof at this point. However, since we believe these Bessel functions to be very significant, we do present the proof of convergence in this most simple case in an appendix at the end of this paper (see Section 8).

2.4. Partial Bessel functions For our purposes, an equally important notion is that of a partial Bessel function. Let $\mathrm{Y}$ be any open compact subgroup of $\mathrm{U}_{w}^{-}$and let $v \in V_{\pi}$ with $W_{v}(e)=1$. Then we define

$$
j_{v, w, Y}(a)=\int_{\mathrm{Y}} W_{v}(a w y) \psi^{-1}(y) d y
$$

again for $a \in \mathrm{A}_{w}$. Note that now there are no convergence problems since $\mathrm{Y}$ is open and compact and $W_{v}$ is smooth (so the integral reduces to a finite sum). Note that if $\left\{\mathrm{Y}_{i}\right\}$ is an increasing exhaustive family of open compact subgroups, then at least formally $J_{\pi, w}=\lim _{i} j_{v, w, Y_{i}}$.

\section{Approximate Whittaker Vectors}

In our finite field heuristics, the vector $v \in V_{\pi}$ which we used to form the Bessel function was a Whittaker vector. Over local fields we do not have Whittaker vectors. However, we can form a family of approximate Whittaker vectors, which we will need, as follows.

Let $\left(\pi, V_{\pi}\right)$ be a generic representation with Whittaker model $\mathcal{W}(\pi)$. Let $v \in V_{\pi}$ be any vector such that $W_{v}(e)=1$. Let $\mathrm{U}_{0} \subset \mathrm{U}(k)$ be an open compact subgroup. Then we set

$$
v_{\mathrm{U}_{0}}=\frac{1}{\operatorname{Vol}\left(\mathrm{U}_{0}\right)} \int_{\mathrm{U}_{0}} \psi^{-1}(u) \pi(u) v d u .
$$

Since the representation is smooth, so that $v$ has a compact open stabilizer, the integration is in fact a finite sum and each $v_{\mathrm{U}_{0}} \in V_{\pi}$. If one considers the 
corresponding Whittaker function, it will satisfy

$$
W_{v_{\mathrm{U}_{0}}}\left(u g u_{0}\right)=\psi(u) W_{v_{\mathrm{U}_{0}}}(g) \psi\left(u_{0}\right)
$$

for $u \in \mathrm{U}$ and $u_{0} \in \mathrm{U}_{0}$. Note that

$$
W_{v_{\mathrm{U}_{0}}}(g)=\frac{1}{\operatorname{Vol}\left(\mathrm{U}_{0}\right)} \int_{\mathrm{U}_{0}} \psi^{-1}(u) W_{v}(g u) d u
$$

so that

$$
W_{v_{\mathrm{U}_{0}}}(e)=W_{v}(e)=1
$$

so that $v_{\mathrm{U}_{0}} \neq 0$.

We will call $v_{\mathrm{U}_{0}}$ an approximate Whittaker vector for $\pi$ with respect to $\mathrm{U}_{0}$.

For any $v \in V_{\pi}$ let $\operatorname{Stab}(v)=\{g \in \mathrm{G}(k) \mid \pi(g) v=v\}$. This is a compact open subgroup of $G(k)$. For $\mathrm{U}_{0}$ a compact open subgroup of $\mathrm{U}(k)$, let

$$
\mathrm{T}_{\mathrm{U}_{0}}=\left\{t \in \mathrm{T}(k) \mid \psi\left(u_{0}\right)=\psi\left(A d(t) u_{0}\right) \quad \text { for all } u_{0} \in \mathrm{U}_{0}\right\} .
$$

Proposition 3.1. Let $v \in V_{\pi}$ such that $W_{v}(e)=1$. Then for any compact open subgroup $\mathrm{U}_{0} \subset \mathrm{U}(k)$ we have

$$
\operatorname{Supp}\left(W_{v_{\mathrm{U}_{0}}}\right) \cap \mathrm{B}(k) \operatorname{Stab}\left(v_{\mathrm{U}_{0}}\right) \subset \mathrm{U}(k) \mathrm{T}_{\mathrm{U}_{0}} \operatorname{Stab}\left(v_{\mathrm{U}_{0}}\right) .
$$

Proof: Let $W_{0}=W_{v_{\mathrm{U}_{0}}}$ and let $\mathrm{K}_{0}=\operatorname{Stab}\left(v_{\mathrm{U}_{0}}\right)$. Let $g=u t k \in \mathrm{B}(k) \mathrm{K}_{0}=$ $\mathrm{U}(k) \mathrm{T}(k) \mathrm{K}_{0}$. Then $W_{0}(u t k)=\psi(u) W_{0}(t)$. Now for any $u_{0} \in \mathrm{U}_{0}$ we have

$$
\psi\left(u_{0}\right) W_{0}(t)=W_{0}\left(t u_{0}\right)=W_{0}\left(t u_{0} t^{-1} t\right)=\psi\left(t u_{0} t^{-1}\right) W_{0}(t) .
$$

Therefore $0=\left(\psi\left(u_{0}\right)-\psi\left(A d(t) u_{0}\right)\right) W_{0}(t)$. Thus $W_{0}(t) \neq 0$ implies $\psi\left(u_{0}\right)=$ $\psi\left(\operatorname{Ad}(t) u_{0}\right)$ for all $u_{0} \in \mathrm{U}_{0}$.

\section{Partial Bessel functions and approximate Whittaker vectors}

In our finite field heuristics in Section 2, the Bessel function was obtained as the Whittaker function associated to a Whittaker vector. In this section we see that we have retained such a relation, at least for the partial Bessel functions and the approximate Whittaker vectors.

Let $w \in W$ be a Weyl group element that supports a Bessel function. For $\mathrm{U}_{*}$ an open compact subgroup of $\mathrm{U}(k)$ we will uniformly assume that $\mathrm{U}_{*}$ satisfies the property

$$
\mathrm{U}_{*}=\mathrm{U}_{*, w}^{+} \mathrm{U}_{*, w}^{-} \quad \text { where } \quad \mathrm{U}_{*, w}^{+}=\mathrm{U}_{*} \cap \mathrm{U}_{w}^{+}, \quad \mathrm{U}_{*, w}^{-}=\mathrm{U}_{*} \cap \mathrm{U}_{w}^{-} .
$$


Accordingly, we will let $\mathrm{Y}_{*}=\mathrm{U}_{*, w}^{-}$, the associated open compact subgroup of $\mathrm{U}_{w}^{-}$. We begin with a proposition which will be useful in the next section as well.

Proposition 4.1. Let $\mathrm{U}_{0} \subset \mathrm{U}_{1}$ be two open compact subsets of $\mathrm{U}(k)$ as above. Let $F(g)$ be a smooth function on $G$ satisfying $F(u g)=\psi(u) F(g)$ for all $u \in \mathrm{U}(k)$, i.e., $F$ is any smooth Whittaker function but not necessarily in the Whittaker model of $\pi$. Let $\chi$ be a smooth function on $G$ satisfying $\chi\left(u g u_{0}\right)=$ $\chi(u)$ for $u \in \mathrm{U}(k)$ and $u_{0} \in \mathrm{U}_{0}$. Let

$$
\tilde{F}(g)=\frac{1}{\operatorname{Vol}\left(\mathrm{U}_{0}\right)} \int_{\mathrm{U}_{0}} F\left(g u_{0}\right) \psi^{-1}\left(u_{0}\right) d u_{0} .
$$

Assume

$$
I(a)=\int_{\mathrm{Y}_{1}} F(a w y) \chi(a w y) \psi^{-1}(y) d y
$$

is convergent for all $a \in \mathrm{A}_{w}$. Then so is

$$
\tilde{I}(a)=\int_{\mathrm{Y}_{1}} \tilde{F}(a w y) \chi(a w y) \psi^{-1}(y) d y
$$

and $I(a)=\tilde{I}(a)$.

Proof: Let $c_{0}=\operatorname{Vol}\left(\mathrm{U}_{0}\right)$. Inserting the definition of $\tilde{F}$ into $\tilde{I}(a)$ we have

$$
\tilde{I}(a)=\frac{1}{c_{0}} \int_{\mathrm{Y}_{1}} \int_{\mathrm{U}_{0}} F\left(a w y u_{0}\right) \psi^{-1}\left(u_{0}\right) d u_{0} \chi(a w y) \psi^{-1}(y) d y .
$$

Interchanging the order of the compact integrations (which are actually finite sums since the functions involved are smooth) we have

$$
\tilde{I}(a)=\frac{1}{c_{0}} \int_{\mathrm{U}_{0}} \int_{\mathrm{Y}_{1}} F\left(a w y u_{0}\right) \chi(a w y) \psi^{-1}\left(y u_{0}\right) d y d u_{0} .
$$

In the inner integral

$$
I_{u_{0}}(a)=\int_{\mathrm{Y}_{1}} F\left(a w y u_{0}\right) \chi(a w y) \psi^{-1}\left(y u_{0}\right) d y
$$

write $u_{0}=u_{0}^{-} u_{0}^{+}$with $u_{0}^{ \pm} \in \mathrm{U}_{0, w}^{ \pm}$. Then we have

$$
I_{u_{0}}(a)=\psi^{-1}\left(u_{0}^{+}\right) \int_{\mathrm{Y}_{1}} F\left(a w y u_{0}^{-} u_{0}^{+}\right) \chi(a w y) \psi^{-1}\left(y u_{0}^{-}\right) d y .
$$


As $\chi$ is left invariant under $\mathrm{U}_{0, w}^{-}$we may perform a change of variables and absorb $u_{0}^{-}$into $y$, leaving

$$
I_{u_{0}}(a)=\psi^{-1}\left(u_{0}^{+}\right) \int_{\mathrm{Y}_{1}} F\left(a w y u_{0}^{+}\right) \chi(a w y) \psi^{-1}(y) d y .
$$

Now, as $\mathrm{U}_{0, w}^{+} \subset \mathrm{U}_{1, w}^{+}$, this normalizes $\mathrm{U}_{1, w}^{-}=\mathrm{Y}_{1}$. Furthermore, as conjugation does not effect the value of the character $\psi(y)$, we may perform a new change of variable in $y$ by conjugating by $u_{0}^{+}$to arrive at

$$
\begin{aligned}
I_{u_{0}}(a) & =\psi^{-1}\left(u_{0}^{+}\right) \int_{\mathrm{Y}_{1}} F\left(a w u_{0}^{+} y\right) \chi(a w y) \psi^{-1}(y) d y \\
& =\psi^{-1}\left(u_{0}^{+}\right) \psi\left(A d(a w) u_{0}^{+}\right) \int_{\mathrm{Y}_{1}} F(a w y) \chi(a w y) \psi^{-1}(y) d y .
\end{aligned}
$$

But since $a \in \mathrm{A}_{w}$ and $\psi$ was chosen to be compatible with the splitting, we have

$$
\psi\left(\operatorname{Ad}(a w) u_{0}^{+}\right)=\psi\left(u_{0}^{+}\right) .
$$

Hence, for each $u_{0}$ we have $I_{u_{0}}(a)=I(a)$. and hence $\tilde{F}(a)=F(a)$.

If we now let $v \in V_{\pi}$ such that $W_{v}(e)=1$ and take $F(g)=W_{v}(g)$ and $\chi \equiv 1$, then we see that $\tilde{F}(g)=W_{v_{0}}(g)$ where $W_{v_{0}}$ is the approximate Whittaker vector associated to $v$ and the open compact subgroup $\mathrm{U}_{0}$. The conclusion of the proposition is then an equality of partial Bessel functions $j_{v, w, \mathrm{Y}_{0}}(a)=$ $j_{v_{0}, w, \mathrm{Y}_{0}}(a)$. On the other hand, since $\mathrm{Y}_{0} \subset \mathrm{U}_{0}$, we see that in this case

$$
\begin{aligned}
j_{v_{0}, w, \mathrm{Y}_{0}}(a) & =\int_{\mathrm{Y}_{0}} W_{v_{0}}(a w y) \psi^{-1}(y) d y=\int_{\mathrm{Y}_{0}} W_{v_{0}}(a w) \psi(y) \psi^{-1}(y) d y \\
& =\operatorname{Vol}\left(\mathrm{Y}_{0}\right) W_{v_{0}}(a w) .
\end{aligned}
$$

Thus we have the following corollary.

Corollary 4.2. For this class of $\mathrm{Y}_{0}$ we always have

$$
j_{v, w, \mathrm{Y}_{0}}(a)=\operatorname{Vol}\left(\mathrm{Y}_{0}\right) W_{v_{0}}(a w) .
$$

So the partial Bessel function is indeed given by the Whittaker function of an approximate Whittaker vector.

As a second corollary, we can investigate how these partial Bessel functions behave as we increase the compact open subgroup. Suppose that $U_{0}$ and $U_{1}$ are compact open subgroups of $\mathrm{U}(k)$ as above with $\mathrm{U}_{0} \subset \mathrm{U}_{1}$. First, taking $F=W_{v_{0}}$ and $\chi \equiv 1$ in Proposition 4.1 and using that since $\mathrm{U}_{0} \subset \mathrm{U}_{1}$ we have $\widetilde{F}=W_{v_{1}}$, we have $j_{v_{0}, w, \mathrm{Y}_{1}}=j_{v, w, \mathrm{Y}_{1}}$. On the other hand, if we begin with $v_{1}$ 
and then compute the partial Bessel function with respect to $\mathrm{U}_{0}$ then automatically $W_{v_{1}}\left(g u_{0}\right)=\psi\left(u_{0}\right) W_{v_{1}}(g)$ and we see $j_{v_{1}, w, \mathrm{Y}_{0}}(a)=\operatorname{Vol}\left(\mathrm{Y}_{0}\right) W_{v_{1}}(a w)=$ $\operatorname{Vol}\left(\mathrm{Y}_{0}\right) \operatorname{Vol}\left(\mathrm{Y}_{1}\right)^{-1} j_{v, w, \mathrm{Y}_{1}}(a)$.

Corollary 4.3. If $\mathrm{U}_{0} \subset \mathrm{U}_{1} \subset \mathrm{U}(k)$ with $\mathrm{U}_{i}$ compact open as above then we have the relations

$$
j_{v_{0}, w, \mathrm{Y}_{1}}=j_{v, w, \mathrm{Y}_{1}} \quad \text { and } \quad j_{v_{1}, w, \mathrm{Y}_{0}}(a)=\left(\mathrm{Y}_{1}: \mathrm{Y}_{0}\right)^{-1} j_{v, w, \mathrm{Y}_{1}}(a)
$$

\section{Asymptotics of Bessel functions I}

We now turn to an investigation of the asymptotics of the Bessel functions attached to a minimal Weyl elements $w$ which supports a Bessel function. From our finite field heuristics, we expect these Bessel function to be supported on the cell $C(w)$, vanish as we approach bounding cells which do not support Bessel functions, and have non-zero asymptotics as we approach the cell $C(e)$ associated to the identity. To investigate the contribution from the other boundary cells, let us number the Weyl elements $w^{\prime}$ such that $C\left(w^{\prime}\right)$ lies on the boundary of $C(w)$ in a convenient fashion. More precisely, let $S_{w}=\left\{w^{\prime} \in\right.$ $\left.W \mid w^{\prime} \leq w\right\}$, let $s=s_{w}=\left|S_{w}\right|$, and enumerate the elements of $S_{w}$ so that $w_{1}^{\prime}=e, w_{s}^{\prime}=w$ and if $w_{i}^{\prime} \leq w_{j}^{\prime}$ then $i \leq j$.

We will be considering various open compact subgroups of $\mathrm{U}(k)$. If we denote one such by $\mathrm{U}_{i}$, we will always work under our standard assumption

$$
\mathrm{U}_{i}=\mathrm{U}_{i, w}^{+} \mathrm{U}_{i, w}^{-} \quad \text { where } \quad \mathrm{U}_{i, w}^{+}=\mathrm{U}_{i} \cap \mathrm{U}_{w}^{+}, \quad \mathrm{U}_{i, w}^{-}=\mathrm{U}_{i} \cap \mathrm{U}_{w}^{-}
$$

and let $\mathrm{Y}_{i}=\mathrm{U}_{i, w}^{-}$be the associated open compact subgroup of $\mathrm{U}_{w}^{-}$with which we form our partial Bessel functions. Similarly, if $v \in V_{\pi}$ with $W_{v}(e)=1$ we will let $v_{i}$ be the associated approximate Whittaker vector

$$
v_{i}=\operatorname{Vol}\left(\mathrm{U}_{i}\right)^{-1} \int_{\mathrm{U}_{i}} \psi^{-1}(u) \pi(u) v d u .
$$

We will also set $j_{i}=j_{v, w, \mathrm{Y}_{i}}=j_{v_{j}, w, \mathrm{Y}_{i}}$ whenever $\mathrm{U}_{j} \subset \mathrm{U}_{i}$.

We begin with two (large) open compact subgroups $\mathrm{U}_{1} \subset \mathrm{U}_{s} \subset \mathrm{U}(k)$ as above. We will fix $\mathrm{U}_{1}$, but $\mathrm{U}_{s}$ will be pushed to be large enough to contain all other $\mathrm{U}_{i}$ constructed. Take $v \in V_{\pi}$ with $W_{v}(e)=1$. Let $\mathrm{K}_{0} \subset \operatorname{Stab}(v)$ be an open compact subgroup of $\mathrm{G}(k)$ fixing $v$. Let

$$
\mathrm{K}_{1}=\bigcap_{u \in \mathrm{U}_{1}} u^{-1} \mathrm{~K}_{0} u .
$$


Since $K_{0} \cap U_{1}$ is compact and open in $U_{1}$ we see that this intersection is in fact finite and $\mathrm{K}_{1}$ is another compact open subgroup of $\mathrm{G}(k)$ which fixes $v$. $\mathrm{B}(k) \mathrm{K}_{1}$ is then a neighborhood of $\mathrm{B}(k) e$ in $\mathrm{B}(k) \backslash \mathrm{G}(k)$.

Let $\chi_{1}$ be the characteristic function of $\mathrm{B}(k) \mathrm{K}_{1}$. Let $H_{1}=W_{v_{1}} \chi_{1}$ and $H_{1}^{\prime}=W_{v_{1}}\left(1-\chi_{1}\right)$. We may accordingly decompose $j_{s}(a)=I_{1}(a)+I_{1}^{\prime}(a)$ for $a \in \mathrm{A}_{w}$, where

$$
I_{1}(a)=\int_{\mathrm{Y}_{1}} H_{1}(a w y) \psi^{-1}(y) d y \quad \text { and } \quad I_{1}^{\prime}(a)=\int_{\mathrm{Y}_{1}} H_{1}^{\prime}(a w y) \psi^{-1}(y) d y .
$$

Proposition 5.1. $I_{1}(a)=\int_{\mathrm{Y}_{s}} W_{v}(a w y) \chi_{1}(a w y) \psi^{-1}(y) d y$.

Proof: This is simply Proposition 4.1 applied to $F=W_{v}, \chi=\chi_{1}$, and $\mathrm{U}_{1} \subset$ $\mathrm{U}_{s}$. We need only check that $\chi_{1}$ satisfies the hypotheses of that proposition. Now $\chi_{1}$ is the characteristic function of $\mathrm{B}(k) \mathrm{K}_{1}$, so the left invariance under $\mathrm{U}(k)$ is clear. Now let $u_{1} \in \mathrm{U}_{1}$. Then $\chi_{1}\left(g u_{1}\right)=1$ iff $g u_{1} \in \mathrm{B}(k) \mathrm{K}_{1}$ iff $g \in$ $\mathrm{B}(k) \mathrm{K}_{1} u_{1}^{-1}$. Since $u_{1} \in \mathrm{U}_{1} \subset \mathrm{B}(k)$, we have $B(k) \mathrm{K}_{1} u^{-1}=\mathrm{B}(k) u_{1} \mathrm{~K}_{1} u_{1}^{-1}=$ $\mathrm{B}(k) \mathrm{K}_{1}$. Hence $\chi_{1}\left(g u_{1}\right)=\chi_{1}(g)$. We can now apply Proposition 4.1.

Note that for $\mathrm{K}_{1}$ sufficiently small, this integral depends only on the behavior of the approximate Whittaker vector near the cell $C(e)$ on the boundary of $C(w)$.

Note first that the function $H_{1}^{\prime}$ satisfies the following properties:

(i) $H_{1}^{\prime}(u g)=\psi(u) H_{1}^{\prime}(g)$ for all $u \in \mathrm{U}(k)$;

(ii) $H_{1}^{\prime}\left(g u_{1}\right)=\psi\left(u_{1}\right) H_{1}^{\prime}(g)$ for all $u_{1} \in \mathrm{U}_{1}$;

(iii) $H_{1}^{\prime}\left(g k_{1}\right)=H_{1}^{\prime}(g)$ for all $k_{1} \in \mathrm{K}_{1}$;

(iv) $\operatorname{Supp}^{\circ}\left(H_{1}^{\prime}\right) \cap \mathrm{B}(k) \mathrm{K}_{1}=\emptyset$.

Here, and throughout, we will let Supp ${ }^{\circ}$ denote the "open support" of a function, i.e., $\operatorname{Supp}^{\circ}(H)=\{g \mid H(g) \neq 0\}$ and so the usual support is given by $\operatorname{Supp}(H)=\overline{\operatorname{Supp}^{\circ}(H)}$.

We would now like to inductively define an increasing sequence of compact open subgroups $\mathrm{U}_{1} \subset \mathrm{U}_{2} \subset \cdots \subset \mathrm{U}_{s-1} \subset \mathrm{U}_{s} \subset \mathrm{U}(k)$, enlarging $\mathrm{U}_{s}$ if necessary, which all satisfy the decomposition properties above, a decreasing sequence compact open subgroups $\mathrm{K}_{1} \supset \mathrm{K}_{2} \supset \cdots \supset \mathrm{K}_{s-1}$, and functions $H_{i}^{\prime}(g)$ for $1 \leq i<s$ such that:

(i) $H_{i}^{\prime}(u g)=\psi(u) H_{i}^{\prime}(g)$ for all $u \in \mathrm{U}(k)$; 
(ii) $H_{i}^{\prime}\left(g u_{i}\right)=\psi\left(u_{i}\right) H_{i}^{\prime}(g)$ for all $u_{i} \in \mathrm{U}_{i}$;

(iii) $H_{i}^{\prime}\left(g k_{i}\right)=H_{i}^{\prime}(g)$ for all $k_{i} \in \mathrm{K}_{i}$;

(iv) $\operatorname{Supp}\left(H_{i}^{\prime}\right) \cap\left(\cup_{j \leq i} C\left(w_{j}^{\prime}\right)\right)=\emptyset$.

Suppose we have constructed $\mathrm{U}_{i}, \mathrm{~K}_{i}$, and $H_{i}^{\prime}$ for $i<j$.

Let $S_{j-1}^{\prime}=\operatorname{Supp}^{\circ}\left(H_{j-1}^{\prime}\right)$. Let $p: \mathrm{G}(k) \rightarrow \mathrm{B}(k) \backslash \mathrm{G}(k)$. Then $p\left(S_{j-1}^{\prime} \cap\right.$ $\left.C\left(w_{j}^{\prime}\right)\right)$ is compact in $p\left(C\left(w_{j}^{\prime}\right)\right)$. Since $p\left(C\left(w_{j}^{\prime}\right)\right)$ is a single $\mathrm{U}(k)$ orbit in $\mathrm{B}(k) \backslash \mathrm{G}(k)$, and in fact homeomorphic to $\mathrm{U}_{w_{j}^{\prime}}^{-}$, there exists a compact open subgroup $\mathrm{U}_{j-1}^{\prime} \subset \mathrm{U}(k)$ such that $p\left(w_{j}^{\prime} \mathrm{U}_{j-1}^{\prime}\right) \supset p\left(S_{j-1}^{\prime}\right) \cap p\left(C\left(w_{j}^{\prime}\right)\right)$. Take $\mathrm{U}_{j}$ large enough so that $\mathrm{U}_{j} \supset\left\langle\mathrm{U}_{j-1}^{\prime}, \mathrm{U}_{j-1}\right\rangle$ and set

$$
\mathrm{K}_{j}=\bigcap_{u_{j} \in \mathrm{U}_{j}} u_{j}^{-1} \mathrm{~K}_{j-1} u_{j} .
$$

As before, this is really a finite intersection.

Consider

$$
\widetilde{H}_{j}(g)=\frac{1}{\operatorname{Vol}\left(\mathrm{U}_{j}\right)} \int_{\mathrm{U}_{j}} H_{j-1}^{\prime}\left(g u_{j}\right) \psi^{-1}\left(u_{j}\right) d u_{j} .
$$

Then $\widetilde{H}_{j}\left(g k_{j}\right)=\widetilde{H}_{j}(g)$ for all $k_{j} \in \mathrm{K}_{j}$.

Let $\widetilde{S}_{j}=\operatorname{Supp}^{\circ}\left(\widetilde{H}_{j}\right)$. Then $p\left(\widetilde{S}_{j}\right) \subset p\left(S_{j}^{\prime} \mathrm{U}_{j}\right)$ and $\overline{p\left(\widetilde{S}_{j}\right)} \cap \overline{p\left(C\left(w_{j}^{\prime}\right)\right)} \subset$ $p\left(w_{j}^{\prime} \mathrm{U}_{j}\right)$. Let $\chi_{j}$ be the characteristic function of $\mathrm{B}(k) w_{j}^{\prime} \mathrm{U}_{j} \mathrm{~K}_{j}$. This set gives a compact neighborhood of $w_{j}^{\prime} \mathrm{U}_{j}$ in $\mathrm{B}(k) \backslash \mathrm{G}(k)$. Then set $H_{j}=\widetilde{H}_{j} \chi_{j}$ and $H_{j}^{\prime}=\widetilde{H}_{j}\left(1-\chi_{j}\right)$. The function $H_{j}^{\prime}$ then satisfies the required properties (i)-(iv) above.

As for the functions $H_{i}$, they satisfy properties similar to those of $H_{1}$, namely:

(a) $H_{i}(u g)=\psi(u) H_{i}(g)$ for all $u \in \mathrm{U}(k)$;

(b) $H_{i}\left(g u_{i}\right)=\psi\left(u_{i}\right) H_{i}(g)$ for all $u_{i} \in \mathrm{U}_{i}$;

(c) $H_{i}\left(g k_{i}\right)=H_{i}(g)$ for all $k_{i} \in \mathrm{K}_{i}$;

(d) $\operatorname{Supp}^{\circ}\left(H_{i}\right) \subset \mathrm{B}(k) w_{i}^{\prime} \mathrm{U}_{i} \mathrm{~K}_{i}$.

However, for $1<i<s$ we have an even stronger property.

Lemma 5.2. If $1<i<s$ and $\mathrm{U}_{i}$ is sufficiently large then $H_{i} \equiv 0$. 
Proof: Since $1<i<s$ the Weyl element $w_{i}^{\prime}$ does not support a Bessel function. By (d) above, $H_{i}(g) \neq 0$ implies that $g \in \mathrm{B}(k) w_{i}^{\prime} \mathrm{U}_{i} \mathrm{~K}_{i}$. Writing $g=u t w_{i}^{\prime} u_{i} k_{i}$ with the implied notation we see $H_{i}\left(u t w_{j}^{\prime} u_{i} k_{i}\right)=\psi\left(u u_{i}\right) H_{i}\left(t w_{i}^{\prime}\right)$. Since $w_{i}^{\prime}$ does not support a Bessel function, there exists a simple root $\alpha \in \Delta$ such that $w_{i}^{\prime} \alpha$ is positive but but not simple. Then for $\mathrm{U}_{i}$ sufficiently large, there exists $u_{i} \in \mathrm{U}_{i} \cap \mathrm{U}_{(\alpha)}$ such that $\psi\left(u_{i}\right) \neq 1$. Then

$$
\psi\left(u_{i}\right) H_{i}\left(t w_{i}^{\prime}\right)=H_{i}\left(t w_{i}^{\prime} u_{i}\right)=H_{i}\left(\left(A d\left(t w_{i}^{\prime}\right) u_{i}\right) t w_{i}^{\prime}\right)=H_{i}\left(t w_{i}^{\prime}\right)
$$

since $A d\left(t w_{i}^{\prime}\right) u_{i} \in \mathrm{U}_{\left(w_{i}^{\prime} \alpha\right)}$. Hence $H_{i}^{\prime}(g)=0$ for $g \in \mathrm{B}(k) w_{i}^{\prime} \mathrm{U}_{i} \mathrm{~K}_{i}$ as well. Hence $H_{i} \equiv 0$.

Now return to the second part of our incomplete Bessel function, namely $I_{1}^{\prime}(a)$. We can similarly define

$$
I_{i}^{\prime}(a)=\int_{\mathrm{Y}_{s}} H_{i}^{\prime}(a w y) \psi^{-1}(y) d y .
$$

Lemma 5.3. For every $1 \leq i<s$ we have $I_{1}^{\prime}(a)=I_{i}^{\prime}(a)$.

Proof: We proceed by finite induction, with the $i=1$ case being true by definition. Suppose we know the lemma for all $j$ with $1 \leq j<i$. Recall that

$$
\widetilde{H}_{i}(g)=\frac{1}{\operatorname{Vol}\left(\mathrm{U}_{i}\right)} \int_{\mathrm{U}_{i}} H_{i-1}^{\prime}\left(g u_{i}\right) \psi^{-1}\left(u_{i}\right) d u_{i}
$$

and $H_{i}^{\prime}(g)=\widetilde{H}_{i}(g) \chi_{i}(g)$. Let us set

$$
\widetilde{I}_{i}(a)=\int_{\mathrm{Y}_{s}} \widetilde{H}_{i}(a w y) \psi^{-1}(y) d y .
$$

If we now apply Proposition 4.1 with $\mathrm{U}_{i} \subset \mathrm{U}_{s}, F=H_{i-1}^{\prime}$ and $\chi \equiv 1$ we have $\widetilde{I}_{i}(a)=I_{i-1}^{\prime}(a)$. On the other hand, since $\widetilde{H}_{i}=H_{i}+H_{i}^{\prime}=H_{i}^{\prime}$ we have $\widetilde{I}_{i}(a)=I_{i}^{\prime}(a)$. Thus, by induction, $I_{i}^{\prime}(a)=I_{i-1}^{\prime}(a)=I_{1}^{\prime}(a)$.

Lemma 5.4. For each $i$ with $1 \leq i<$ s let us set $\chi_{i}^{\prime}=\prod_{1 \leq j \leq i}\left(1-\chi_{j}\right)$. Let $\tilde{\chi}$ be a function on $\mathrm{G}(k)$ such that $\widetilde{\chi}\left(u g u_{i}\right)=\widetilde{\chi}(g)$ for all $u \in \mathrm{U}(k)$ and $u_{i} \in \mathrm{U}_{i}$. Then

$$
I_{i}^{\prime}(a)=\int_{\mathrm{Y}_{s}} W_{v}(a w y) \chi_{i}^{\prime}(a w y) \psi^{-1}(y) d y .
$$

Proof: For each $i$, let $\widetilde{\chi}_{i}$ be a function on $\mathrm{G}(k)$ such that $\widetilde{\chi}\left(u g u_{i}\right)=\widetilde{\chi}(g)$ for all $u \in \mathrm{U}(k)$ and $u_{i} \in \mathrm{U}_{i}$. Let

$$
I_{i}^{\prime}\left(a, \widetilde{\chi}_{i}\right)=\int_{\mathrm{Y}_{s}} H_{i}^{\prime}(a w y) \widetilde{\chi}_{i}(a w y) \psi^{-1}(y) d y
$$


so that we obtain $I_{i}^{\prime}(a)$ itself by taking $\widetilde{\chi}_{i} \equiv 1$.

By induction, we will show that

$$
I_{i}^{\prime}\left(a, \widetilde{\chi}_{i}\right)=\int_{\mathrm{Y}_{s}} W_{v}(a w y) \chi_{i}^{\prime}(a w y) \widetilde{\chi}_{i}(a w y) \psi^{-1}(y) d y .
$$

In the case $i=1$, then by definition

$$
\begin{aligned}
I_{1}^{\prime}\left(a, \widetilde{\chi}_{1}\right) & =\int_{\mathrm{Y}_{s}} H_{1}^{\prime}(a w y) \widetilde{\chi}_{1}(a w y) \psi^{-1}(y) d y \\
& =\int_{\mathrm{Y}_{s}} W_{v_{1}}(a w y)\left(1-\chi_{1}(a w y)\right) \widetilde{\chi}_{1}(a w y) \psi^{-1}(y) d y
\end{aligned}
$$

and the result follows from Proposition 4.1.

Now suppose the statement is true for all $j$ with $1 \leq j<i$. Then

$$
\begin{aligned}
I_{i}^{\prime}\left(a, \widetilde{\chi}_{i}\right) & =\int_{\mathrm{Y}_{s}} H_{i}^{\prime}(a w y) \widetilde{\chi}_{i}(a w y) \psi^{-1}(y) d y \\
& =\int_{\mathrm{Y}_{s}} \widetilde{H}_{i}(a w y)\left(1-\chi_{i}(a w y)\right) \widetilde{\chi}_{i}(a w y) \psi^{-1}(y) d y .
\end{aligned}
$$

Now apply Proposition 4.1 with $\mathrm{U}_{i} \subset \mathrm{U}_{l}, F=H_{i-1}^{\prime}$ and $\chi=\left(1-\chi_{i}\right) \tilde{\chi}_{i}$. We then obtain

$$
I_{i}^{\prime}\left(a, \widetilde{\chi}_{i}\right)=\int_{\mathrm{Y}_{s}} H_{i-1}^{\prime}(a w y)\left(1-\chi_{i}(a w y)\right) \tilde{\chi}_{i}(a w y) \psi^{-1}(y) d y .
$$

Now we apply our induction hypothesis with $\tilde{\chi}_{i-1}=\left(1-\chi_{i}\right) \tilde{\chi}_{i}$ to obtain

$$
\begin{aligned}
I_{i}^{\prime}\left(a, \tilde{\chi}_{i}\right) & =\int_{\mathrm{Y}_{s}} W_{v}(a w y) \chi_{i-1}^{\prime}(a w y)\left(1-\chi_{i}(a w y)\right) \tilde{\chi}_{i}(a w y) \psi^{-1}(y) d y \\
& =\int_{\mathrm{Y}_{s}} W_{v}(a w y) \chi_{i}^{\prime}(a w y) \widetilde{\chi}_{i}(a w y) \psi^{-1}(y) d y .
\end{aligned}
$$

Now, applying this with $\tilde{\chi}_{i} \equiv 1$ gives the desired statement.

Recall that we had decomposed $j_{s}(a)$ as $j_{s}(a)=I_{1}(a)+I_{1}^{\prime}(a)$ As a consequence of these lemmas we may write

$$
I_{1}^{\prime}(a)=I_{s-1}^{\prime}(a)=\int_{\mathrm{Y}_{s}} W_{v}(a w y) \chi_{s-1}^{\prime}(a w y) \psi^{-1}(y) d y
$$

where $\chi_{s-1}^{\prime}=\prod_{1 \leq j \leq s-1}\left(1-\chi_{j}\right)$. Since $\chi_{j}$ was the characteristic function of $\mathrm{B}(k) w_{j}^{\prime} \mathrm{U}_{j} \mathrm{~K}_{j}$ we see that $\operatorname{Supp}\left(\chi_{s-1}^{\prime}\right)$ is the characteristic function of the compliment of

$$
\bigcup_{1 \leq j<s} \mathrm{~B}(k) w_{j}^{\prime} \mathrm{U}_{j} \mathrm{~K}_{j}
$$


and by construction this is a neighborhood of

$$
\partial C(w)=\bigcup_{1 \leq j<s} C\left(w_{j}^{\prime}\right) .
$$

Hence there will be an open compact neighborhood $\Omega$ of $e$ in $\mathrm{U}_{w}^{-}$such that $C(w) \cap \operatorname{Supp}\left(\chi_{s-1}^{\prime}\right)=\mathrm{B}(k) w \Omega$. If we then let $\chi_{\Omega}$ denote the characteristic function of $\Omega$ we have

$$
I_{1}^{\prime}(a)=\int_{\mathrm{Y}_{s}} W_{v}(a w y) \chi_{\Omega}(y) \psi^{-1}(y) d y .
$$

Note that $\Omega$ does not depend on our choice of $\mathrm{U}_{s}$. Hence choosing $\mathrm{U}_{s}$ sufficiently large, we can assume that $\Omega \subset \mathrm{U}_{s, w}^{-}=\mathrm{Y}_{s}$.

Thus we have established the following proposition.

Proposition 5.5. Let $w$ be a minimal Weyl element supporting a Bessel function and let $v \in V_{\pi}$ with $W_{v}(e)=1$. Then there exist a compact open subgroup $\mathrm{K}_{1}$ of $\mathrm{G}(k)$ and a compact open neighborhood $\Omega$ of $e$ in $\mathrm{U}_{w}^{-}$such that for every sufficiently large compact open subgroup $\mathrm{U}_{s}$ of $U(k)$

$$
j_{v, w, \mathrm{Y}_{s}}(a)=\int_{\mathrm{Y}_{s}} W_{v}(a w y) \chi_{1}(a w y) \psi^{-1}(y) d y+\int_{\Omega} W_{v}(a w y) \psi^{-1}(y) d y
$$

where $\chi_{1}$ is the characteristic function of $\mathrm{B}(k) \mathrm{K}_{1}$.

As expected, this gives that there are two contributions to the Bessel function associated to $w$-one from the cell $C(w)$ associated to $w$ itself and one from $C(e)$, the only cell on the boundary of $C(w)$ that supports a Bessel function.

As a corollary, note that since $v$ is a smooth vector of $V_{\pi}$ and $\Omega$ is compact then

$$
v_{\Omega}=\int_{\Omega} \psi^{-1}(y) \pi(y) v d y
$$

is a finite sum, so that $v_{\Omega} \in V_{\pi}$ and

$$
\int_{\Omega} W_{v}(a w y) \psi^{-1}(y) d y=W_{v_{\Omega}}(a w) .
$$

This then gives the following corollary.

Corollary 5.6. Let $w$ be a minimal Weyl element supporting a Bessel function and let $v \in V_{\pi}$ with $W_{v}(e)=1$. Then there exist a compact open subgroup $\mathrm{K}_{1}$ of $G(k)$ and a vector $v_{\Omega} \in V_{\pi}$ such that for every sufficiently large compact 
open subgroup $\mathrm{U}_{s}$ of $U(k)$

$$
j_{v, w, \mathrm{Y}_{s}}(a)=\int_{\mathrm{Y}_{s}} W_{v}(a w y) \chi_{1}(a w y) \psi^{-1}(y) d y+W_{v_{\Omega}}(a w)
$$

where $\chi_{1}$ is the characteristic function of $\mathrm{B}(k) \mathrm{K}_{1}$.

\section{The contribution from near $C(e)$}

For applications functoriality as in $[\mathbf{8}, \mathbf{6}, \mathbf{7}]$ it is essential that we be able to show that the first integral occurring in the expression of the Bessel function in Corollary 5.6 - the contribution from the cell $C(e)$ - is only mildly dependent on the representation $\pi$. In fact, it will depend only on the central character $\omega_{\pi}$ of $\pi$. For this purpose it is easier if we take $\mathrm{U}_{1}$ to be one of the $\mathrm{U}_{f_{M}}$ of Section 1.1. This is clearly permissible since this family of compact open subgroups is cofinal and satisfy the decomposition properties of Section 4 . (In fact, we could have taken each $\mathrm{U}_{j}$ as $\mathrm{U}_{f_{M_{j}}}$ with $M=M_{1} \leq M_{2} \leq \cdots \leq M_{s}$.)

We are interested in the first term

$$
I_{1}(a)=\int_{\mathrm{Y}_{s}} W_{v}(a w y) \chi_{1}(a w y) \psi^{-1}(y) d y
$$

of Corollary 5.6, which by Proposition 5.1 we know is equal to

$$
I_{1}(a)=\int_{\mathrm{Y}_{s}} W_{v_{1}}(a w y) \chi_{1}(a w y) \psi^{-1}(y) d y
$$

with

$$
v_{1}=\operatorname{Vol}\left(\mathrm{U}_{1}\right)^{-1} \int_{\mathrm{U}_{1}} \psi^{-1}(u) \pi(u) v d u
$$

Recall that $\chi_{1}$ is the characteristic function of $\mathrm{B}(k) \mathrm{K}_{1}$ with $\mathrm{K}_{1}$ open and compact. It is easy to see that, by construction, $\mathrm{K}_{1} \subset \operatorname{Stab}\left(v_{1}\right)$. Then applying Proposition 3.1 we see that the support of $W_{v_{1}} \chi_{1}$ is contained in $\mathrm{U}(k) \mathrm{T}_{\mathrm{U}_{1}} \operatorname{Stab}\left(v_{1}\right)$.

Consider now $\mathrm{T}_{\mathrm{U}_{1}}$. By definition,

$$
\mathrm{T}_{\mathrm{U}_{1}}=\left\{t \in \mathrm{T}(k) \mid \psi\left(u_{1}\right)=\psi\left(A d(t) u_{1}\right) \quad \text { for all } u_{1} \in \mathrm{U}_{1}\right\} .
$$

Now take $\mathrm{U}_{1}=\mathrm{U}_{f_{M}}$. We have seen in Section 1.2 that we can write our generic character as

$$
\psi(u)=\psi\left(\sum_{\alpha \in \Delta} \bar{x}_{\alpha}\left(u_{\tilde{\alpha}}\right)\right)=\prod_{\alpha \in \Delta} \psi\left(\operatorname{Tr}_{k_{\tilde{\alpha}} / k}\left(u_{\tilde{\alpha}}\right)\right),
$$


or, letting $\psi_{\alpha}=\psi \circ \operatorname{Tr}_{K_{\tilde{\alpha}} / k}$, we have $\psi=\prod \psi_{\alpha}$ under the isomorphism $\mathrm{U}^{a b} \simeq \oplus \mathrm{R}_{K_{\tilde{\alpha}} / k} \mathbb{G}_{a}$. Thus, the condition that $t$ lies in $\mathrm{T}_{\mathrm{U}_{1}}$ becomes

$$
\psi_{\alpha}\left(u_{\tilde{\alpha}}-\tilde{\alpha}(t) u_{\tilde{\alpha}}\right)=1
$$

for all $u_{\tilde{\alpha}} \in K_{\tilde{\alpha}}$ with $\omega\left(u_{\tilde{\alpha}}\right) \geq-M$. If we normalize our additive character of $k$ in such a way that it is trivial on $\mathcal{O}$ but not on $\mathcal{P}^{-1}$, i.e., $\psi(u)=1$ for $\omega(u) \geq 0$ but there exists $u$ with $\omega(u)=-1$ such that $\psi(u) \neq 1$, then $\psi_{\alpha}\left(u_{\tilde{\alpha}}\right)=1$ for $\omega\left(u_{\tilde{\alpha}}\right) \geq-d_{\tilde{\alpha}}$ where $\mathcal{P}_{\tilde{\alpha}}^{-d_{\tilde{\alpha}}}$ is the inverse different of $K_{\tilde{\alpha}}$. Thus our condition that $t$ lie in $\mathrm{T}_{\mathrm{U}_{1}}$ becomes

$$
\omega\left((1-\tilde{\alpha}(t)) u_{\tilde{\alpha}}\right) \geq-d_{\tilde{\alpha}}
$$

for all $u_{\tilde{\alpha}}$ with $\omega\left(u_{\tilde{\alpha}}\right) \geq-M$, i.e.,

$$
\tilde{\alpha}(t) \in 1+\mathcal{P}_{\tilde{\alpha}}^{M-d_{\tilde{\alpha}}} \text { for all } \quad \tilde{\alpha} \in \widetilde{\Delta} .
$$

Recalling the notation of Section 1.3, and writing $d_{i}=d_{\tilde{\alpha}_{i}}$, we have established the following lemma.

Lemma 6.1. Suppose $\mathrm{U}_{1}=\mathrm{U}_{f_{M}}$ with $M$ sufficiently large. Then for $\underline{M}=$ $(M, \ldots, M)-\left(d_{1}, \ldots, d_{r}\right)$ we have

$$
\mathrm{T}_{\mathrm{U}_{1}}=\mathrm{T}_{\underline{M}}=\mathrm{Z}(k) \mathrm{T}_{\underline{M}}^{1} .
$$

Proof: The first equality follows from the argument preceeding the statement of the lemma. The second equality then follows from Lemma 1.1 for $M$ sufficiently large.

Return now to our expression (6.2) for the contribution to the Bessel function from the small cell $C(e)$. Then the support of $W_{v_{1}} \chi_{1}$ is contained in $\mathrm{U}(k) \mathrm{T}_{\mathrm{U}_{1}} \mathrm{~K}_{1}$ with $\mathrm{K}_{1} \subset \operatorname{Stab}\left(v_{1}\right)$, and we can now write this as $\mathrm{U}(k) \mathrm{Z}(k) \mathrm{T}_{\underline{M}}^{1} \mathrm{~K}_{1}$, taking $\mathrm{U}_{1}=\mathrm{U}_{f_{M}}$. Let us write this decomposition as

$$
g=u(g) z(g) t^{1}(g) k_{1}(g) \quad \text { for } \quad g \in \mathrm{U}(k) \mathrm{Z}(k) \mathrm{T}_{\underline{M}}^{1} \mathrm{~K}_{1} .
$$

Then we can write

$$
W_{v_{1}}(a w y) \chi_{1}(a w y)=\psi(u(a w y)) \omega_{\pi}(z(a w y)) W_{v_{1}}\left(t^{1}(a w y)\right)
$$

for $a w y \in \mathrm{U}(k) \mathrm{Z}(k) \mathrm{T}_{\underline{M}}^{1} \mathrm{~K}_{1}$, or $y \in(a w)^{-1} \mathrm{U}(k) \mathrm{Z}(k) \mathrm{T}_{\underline{M}}^{1} \mathrm{~K}_{1}$.

For $\mathrm{U}_{1}=\mathrm{U}_{f_{M}}$ we have that

$$
v_{1}=\operatorname{Vol}\left(\mathrm{U}_{f_{M}}\right)^{-1} \int_{\mathrm{U}_{f_{M}}} \psi^{-1}(u) \pi(u) v d u .
$$


Then if $t^{1} \in \mathrm{T}_{\underline{M}}^{1}$ we see that

$$
\pi\left(t^{1}\right) v_{1}=\operatorname{Vol}\left(\mathrm{U}_{f_{M}}\right)^{-1} \int_{\mathrm{U}_{f_{M}}} \psi^{-1}\left(A d\left(t^{1}\right) u\right) \pi(u) \pi\left(t^{1}\right) v d u .
$$

Since $\mathrm{T}_{\underline{M}}^{1} \subset \mathrm{T}_{\underline{M}}=\mathrm{T}_{\mathrm{U}_{1}}$ we see that

$$
\psi\left(A d\left(t^{1}\right) u\right)=\psi(u)
$$

for all $u \in \mathrm{U}_{1}=\mathrm{U}_{f_{M}}$. Hence we see that if $M$ is sufficiently large that $\mathrm{T}_{\underline{M}}^{1} \subset \operatorname{Stab}(v)$, then $\mathrm{T}_{\underline{M}}^{1} \subset \operatorname{Stab}\left(v_{1}\right)$ so that

$$
W_{v_{1}}\left(t^{1}(a w y)\right)=W_{v_{1}}(e)=W_{v}(e)=1 .
$$

Note that the vector $v$ was fixed at the beginning of our construction and precedes any other choices made. Hence we see that if $M$ is taken sufficiently large we have the support of $W_{v_{1}} \chi_{1}$ is precisely $\mathrm{U}(k) \mathrm{Z}(k) \mathrm{T}_{\underline{M}}^{1} \mathrm{~K}_{1}$.

Combining these analyses, we arrive at the following proposition.

Proposition 6.2. Let $v \in V_{\pi}$ be such that $W_{v}(e)=1$. Choose $M$ sufficiently large so that $\mathrm{T}_{\underline{M}}^{1} \subset \mathrm{Stab}(v)$ and that Lemma 1.1 holds. Take $\mathrm{U}_{1}=\mathrm{U}_{f_{M}}$. Then for $a \in \mathrm{A}_{w}$ we have

$$
\begin{aligned}
I_{1}(a) & =\int_{\mathrm{Y}_{s}} W_{v}(a w y) \chi_{1}(a w y) \psi^{-1}(y) d y \\
& =\int_{\mathrm{Y}_{s} \cap(a w)^{-1} \mathrm{U}(k) \mathrm{Z}(k) \mathrm{T}_{\underline{M}}^{1} \mathrm{~K}_{1}} \psi(u(a w y)) \omega_{\pi}(z(a w y)) \psi^{-1}(y) d y
\end{aligned}
$$

which depends on the representation $\pi$ only through its central character $\omega_{\pi}$.

\section{Asymptotics of Bessel functions II}

Let us now return to our partial Bessel function $j_{v, w, Y_{s}}(a)$ from Corollary 5.6

$$
j_{v, w, Y_{s}}(a)=\int_{\mathrm{Y}_{s}} W_{v}(a w y) \chi_{1}(a w y) \psi^{-1}(y) d y+W_{v_{\Omega}}(a w) .
$$

Recall that the first term, namely $I_{1}(a)$ from $(6.1)$, is the contribution to the Bessel function from near the small cell $C(e)$ while the second term $W_{v_{\Omega}}(a w)$ is the contribution from the "interior" of the cell $C(w)$.

Consider first the contribution from $C(w)$. We may rewrite this as

$$
j_{v, w, Y_{s}}^{C(w)}(a)=W_{v_{\Omega}}(a w)=W_{\pi(w) v_{\Omega}}(a) .
$$


In this form it is seen to be given by the value of a Whittaker function for a vector $\pi(w) v_{\Omega} \in V_{\pi}$. As a function of $a \in \mathrm{A}_{w}$ this then is compactly supported as $\alpha(a) \rightarrow \infty$ for simple roots $\alpha \in \Delta$, as all smooth Whittaker functions are, and satisfies asymptotics determined by the representation $\pi$ as $\alpha(a) \rightarrow 0$. Hence its asymptotics are well understood and are determined by the representation $\pi$. Also, since this contribution is given by a fixed Whittaker function, it is smooth as a function of $a \in \mathrm{A}_{w}$.

The contribution from the cell $C(e)$, which we will now denote by $j_{v, w, \mathrm{Y}_{s}}^{C(e)}(a)$, is quite interesting and was analyzed in the last section. From Proposition 6.2 we know that $j_{v, w, Y_{s}}^{C(e)}(a)=I_{1}(a)$ is actually given by a quite complicated exponential sum, namely

$$
j_{v, w, \mathrm{Y}_{s}}^{C(e)}(a)=\int_{\mathrm{Y}_{s} \cap(a w)^{-1} \mathrm{U}(k) \mathrm{Z}(k) \mathrm{T}_{\underline{M}}^{1} \mathrm{~K}_{1}} \psi(u(a w y)) \omega_{\pi}(z(a w y)) \psi^{-1}(y) d y .
$$

Since the contribution from $C(w)$ vanishes as $\alpha(a) \rightarrow \infty$ we see that the asymptotics of the Bessel function $j_{v, w, \mathrm{Y}_{s}}(a)$ as $\alpha(a) \rightarrow \infty$ are completely given by this exponential sum. Fortunately for our application, even though this sum is complicated, it depends on the representation $\pi$ only through its central character $\omega_{\pi}$.

Combining the above, we finally arrive at the main theorem of this paper.

Theorem 7.1. Let $\left(\pi, V_{\pi}\right)$ be a generic representation of $\mathrm{G}(k)$ and let $v \in V_{\pi}$ satisfy $W_{v}(e)=1$. Let $w \in W$ be a Weyl group element which supports a Bessel function and is a minimal non-trivial such. Then for every sufficiently large compact open subgroup $\mathrm{Y} \subset \mathrm{U}_{w}^{-}(k)$ we have that the partial Bessel function $j_{v, w, \mathrm{Y}}(a)$ can be decomposed as

$$
j_{v, w, Y}(a)=j_{v, w, Y}^{C(e)}(a)+j_{v, w, Y}^{C(w)}(a)
$$

where $j_{v, w, \mathrm{Y}}^{C(w)}(a)$ is given by (7.1) and is a smooth function of $a \in \mathrm{A}_{w}$ which vanishes as $\alpha(a) \rightarrow \infty$ and $j_{v, w, \mathrm{Y}}^{C(e)}(a)$ is given by (7.2) and is dependent only on the central character $\omega_{\pi}$ of $\pi$.

We will return to the application of this result to the stability of local $\gamma$-functions for generic representations of quasi-split groups in a subsequent paper [9]. 


\section{Appendix: On the existence of $J_{\pi, w}(a)$.}

In this Appendix we will present the arguments for the convergence of the Bessel function $J_{\pi, w}(a)$ attached to a minimal Weyl element $w$ which supports a Bessel function from Section 2.3. We begin with some preliminaries which generalize a construction of Steinberg [17] to the quasi-split setting.

8.1. Coefficients of Steinberg Let $\mathfrak{g}$ denote the Lie algebra of G. Recall [2] that we have the decomposition of the Lie algebra of $\mathrm{G}$ given by

$$
\mathfrak{g}=\mathfrak{g}_{0} \oplus \bigoplus_{\alpha \in \Phi_{n d}} \mathfrak{g}_{(\alpha)}
$$

For each non-divisible root $\alpha \in \Phi_{n d}$ chose a $k$-rational basis $\left\{X_{\alpha, i_{\alpha}} \mid 1 \leq\right.$ $\left.i_{\alpha} \leq \operatorname{dim}\left(\mathfrak{g}_{(\alpha)}\right)\right\}$ of $\mathfrak{g}_{(\alpha)}$. Similarly choose a $k$-rational basis $\left\{H_{1}, \ldots, H_{r}\right\}$ of $\mathfrak{g}_{0}=\mathfrak{t}$, the Lie algebra of the maximal $k$-torus $T$. We may assume this basis is an extension of a basis of $\mathfrak{a}$, the Lie algebra of $\mathrm{A}$.

Let $N=\operatorname{dim}_{k} \mathfrak{u}$ be the dimension of the maximal unipotent subgroup $\mathrm{U}$ of $\mathrm{G}$, or its Lie algebra $\mathfrak{u}$. Consider the $N^{\text {th }}$ exterior product of $\mathfrak{g}, \wedge^{N} \mathfrak{g}$ with the basis consisting of wedge products of the basis $\left\{X_{\alpha, i_{\alpha}}, H_{j}\right\}$ of $\mathfrak{g}$. G acts $k$-rationally on $\mathfrak{g}$, and hence on $\wedge^{N} \mathfrak{g}$, by the adjoint action.

Let

$$
Y_{e}=\wedge_{\alpha>0} \wedge_{i_{\alpha}} X_{\alpha, i_{\alpha}}
$$

This is one of our canonical basis vectors. Similarly, for any $w \in W$, let

$$
Y_{w}=\wedge_{\alpha>0} \wedge_{i_{w \alpha}} X_{w \alpha, i_{w \alpha}} .
$$

For any $g \in G$ define $c_{w}(g)$ to be the coefficient of $Y_{w}$ in the expansion of $A d(g) Y_{e}$ in our chosen basis for $\wedge^{N} \mathfrak{g}$. Since the adjoint action is $k$-rational, this is a well defined rational function of $g$. Note that $A d(w) Y_{e}=c_{w}(w) Y_{w}$ with $c_{w}(w) \neq 0$.

From the relation $\left[\mathfrak{g}_{\alpha}, \mathfrak{g}_{\beta}\right] \subset \mathfrak{g}_{\alpha+\beta}$ it follows that for $u \in \mathrm{U}$ we have $A d(u) Y_{e}=c_{e}(u) Y_{e}$ with $c_{e}(u) \neq 0$. Then for $t \in \mathrm{T}$ we also have $\operatorname{Ad}(t) Y_{e}=$ $c_{e}(t) Y_{e}$ with $c_{e}(t) \neq 0$ being essentially the modulus character of $t$. As noted above, for $w \in W$ we have $A d(w) Y_{e}=c_{w}(w) Y_{w}$ by definition. Finally, if $u \in \mathrm{U}$ we have $A d(u) Y_{w}=c Y_{w}+$ "higher order terms", with respect to the ordering given by sums of positive roots, with $c \neq 0$. Thus $A d(\mathrm{~B} w \mathrm{~B}) Y_{e} \subset k^{\times} Y_{w}+$ "higher order terms", i.e., $c_{w}$ is non-vanishing on the Bruhat cell BwB. Then the proof of Theorem 23 of Steinberg [17] (p.127) gives the following result. 
Proposition 8.1. Let $w, w^{\prime} \in W$. Then the following three conditions are equivalent:

(i) $\mathrm{B} w^{\prime} \mathrm{B} \subset \overline{\mathrm{B} w \mathrm{~B}}$;

(ii) $w^{\prime} \leq w$

(iii) $c_{w^{\prime}}(g)$ is not identically zero on $\mathrm{B} w \mathrm{~B}$.

Note that as a consequence of this, we see that for $w=w_{\ell}$ the long Weyl element we have that $c_{w_{\ell}}$ is non-vanishing precisely on the big Bruhat cell $C\left(w_{\ell}\right)$. Moreover, Lemma 52 of Steinberg [17] (p.123), generalized to the quasi-split situation, gives that $c_{e}$ is non-vanishing precisely on the translated large cell $\mathrm{U}^{-} \mathrm{TU}=w_{\ell}^{-1} C\left(w_{\ell}\right)$. To compute the support of the other coefficient functions, we have the following lemma.

Lemma 8.2. For any $w \in W$,

$$
c_{w}(w g)=c_{e}(g) c_{w}(w)
$$

Proof: This is an elementary calculation. On the one hand, by definition we have $A d(w g) Y_{e}=c_{w}(w g) Y_{w}+\cdots$. On the other hand,

$$
\begin{aligned}
A d(w g) Y_{e} & =A d(w) A d(g) Y_{e}=A d(w)\left(c_{e}(g) Y_{e}+\cdots\right) \\
& =c_{e}(g) c_{w}(w) Y_{w}+\cdots
\end{aligned}
$$

Thus $c_{w}(w g)=c_{e}(g) c_{w}(w)$.

An equivalent formulation is that $c_{w}(g)=c_{e}\left(w^{-1} g\right) c_{w}(w)$. Thus we have $c_{w}(g) \neq 0$ iff $c_{e}\left(w^{-1} g\right) \neq 0$ iff $w^{-1} g \in \mathrm{U}^{-} \mathrm{B}$ iff $g \in w \mathrm{U}^{-} \mathrm{B}=w w_{\ell}^{-1} \mathrm{U} w_{\ell} \mathrm{B}$.Thus we have the following generalization of the above statements.

Proposition 8.3. $c_{w}(g)$ is non-vanishing precisely on $w^{-} \mathrm{B}$.

The above lemma also lets us explicitly evaluate the Steinberg coefficients. First recall that for $t \in \mathrm{T}$ we have the modulus character $\delta(t)=\delta_{\mathrm{B}}(t)=$ $\operatorname{det}(A d(t) \mid \mathrm{U})$. Then we see that by definition $c_{e}(t)=\delta(t)$. Hence on the Bruhat cell $C(e)=\mathrm{B}$ we have $c_{e}(t u)=\delta(t) \neq 0$, as claimed. Next consider $c_{w}$ as a function on $C(w)$ If we write $g \in C(w)$ as $g=u_{1} w t u_{2}$ we see that

$$
\begin{aligned}
A d\left(u_{1} w t u_{2}\right) Y_{e} & =A d\left(u_{1} w t\right) Y_{e}=A d\left(u_{1} w\right) c_{e}(t) Y_{e} \\
& =c_{e}(t) A d\left(u_{1}\right) c_{w}(w) Y_{w}=c_{e}(t) c_{w}(w) Y_{w}+\text { "higher order terms" }
\end{aligned}
$$

since $u_{1} \in \mathrm{U}(k)$. Thus $c_{w}\left(u_{1} w t u_{2}\right)=\delta(t) c_{w}(w)$.

For our purposes, a more useful formula will be $c_{w}\left(u_{1} t w u_{2}\right)=c_{w}(w) \delta\left(w^{-1} t w\right)$. 
Let us set $\delta_{w}(t)=\delta\left(w^{-1} t w\right)=\operatorname{det}\left(A d(t) \mid w \mathrm{U} w^{-1}\right)$ the modulus character associated to the conjugate Borel $\mathrm{B}^{w}=w \mathrm{~B} w^{-1}$.

Proposition 8.4. Let $w \in W$ and let $S(w t)$ denote the slice $\mathrm{U}(k) w t \mathrm{U}(k)$ of the Bruhat cell $C(w)$. Then $c_{w}$ is constant on this slice and $c_{w}(S(t w))=$ $\delta(t) c_{w}(w)$. Moreover, $c_{w}$ is constant on the slice $S(t w)$ of $C(w)$ and on this slice $c_{w}(S(t w))=\delta_{w}(t) c_{w}(w)$.

8.2. The convergence of $J_{\pi, w}(a)$ We now return to the situation of Sections 2 and 3 .

Proposition 8.5. Let $v \in V_{\pi}$ such that $W_{v}(e)=1$. For any compact open subgroup $\mathrm{U}_{0} \subset \mathrm{U}(k)$ let $v_{0}=v_{\mathrm{U}_{0}}$ be the associated approximate Whittaker vector in $V_{\pi}$. Let $w \in W, w \neq e$. For $t \in \mathrm{T}(k)$ consider the slice $S(t w)=$ $\mathrm{U}(k) t w \mathrm{U}(k)=\mathrm{U}(k) t w \mathrm{U}_{w}^{-}$of the Bruhat cell $C(w)=\mathrm{B} w \mathrm{U}_{w}^{-}$. Then there is an open compact subgroup $\mathrm{K}_{0}^{\prime}$ such that the restriction of $W_{v_{0}}$ to $S(t w)$ vanishes on $S(t w) \cap \mathrm{BK}_{0}^{\prime}$.

Proof: Let $W_{0}=W_{v_{0}}$ and let $\mathrm{K}_{0}=\operatorname{Stab}\left(v_{0}\right)$. If $\mathrm{K}^{\prime}$ is any subgroup of $\mathrm{K}_{0}$ then as in the proof of the Proposition 3.1 we have that $\operatorname{Supp}\left(W_{0}\right) \cap \mathrm{B}(k) \mathrm{K}^{\prime} \subset$ $\mathrm{U}(k) \mathrm{T}_{\mathrm{U}_{0}} \mathrm{~K}^{\prime}$. Hence the proposition will follow if, given $w \neq e$ and $t \in \mathrm{T}(k)$, we can find an open neighborhood $\mathrm{K}_{0}^{\prime}$ of $e$ such that $S(t w) \cap \mathrm{U}(k) \mathrm{T}_{\mathrm{U}_{0}} \mathrm{~K}_{0}^{\prime}=\emptyset$. Taking inverses, it suffices to find a $\mathrm{K}_{0}^{\prime}$ such that $S\left(w^{-1} t^{-1}\right) \cap \mathrm{K}_{0}^{\prime} \mathrm{T}_{\mathrm{U}_{0}} \mathrm{U}(k)=\emptyset$.

Recall from Section 8.1 that to any $w \in W$ there is associated a rational (hence continuous) function $c_{w}(g)$ such that $C(w) \subset \overline{C\left(w^{\prime}\right)}$ iff $c_{w}(g)$ is not identically zero on $C\left(w^{\prime}\right)$. In particular, for $w \neq e$ we have $c_{w}(b)=0$ for all $b \in \mathrm{B}(k)=C(e)$. On the other hand, we have seen that $c_{w}(g)$ is a non-zero constant on either of the slices $S(w t)=\mathrm{U}(k) w t \mathrm{U}(k)$ or $S(t w)$.

Now consider the restriction of $c_{w^{-1}}$ to the open set $\mathrm{K}^{\prime} \mathrm{T}_{\mathrm{U}_{0}} \mathrm{U}(k)$. Then for $k^{\prime} t^{\prime} u \in \mathrm{K}^{\prime} \mathrm{T}_{\mathrm{U}_{0}} \mathrm{U}(k)$ we have $A d\left(k^{\prime} t^{\prime} u\right) Y_{e}=c_{e}\left(t^{\prime}\right) A d\left(k^{\prime}\right) Y_{e}$ so that $c_{w^{-1}}\left(k^{\prime} t^{\prime} u\right)=$ $c_{e}\left(t^{\prime}\right) c_{w^{-1}}\left(k^{\prime}\right)$. Since $t^{\prime} \in \mathrm{T}_{\mathrm{U}_{0}}$ satisfies $\psi\left(u_{0}\right)=\psi\left(A d\left(t^{\prime}\right) u_{0}\right)$ for all $u_{0}$ in the compact open subgroup $\mathrm{U}_{0}$ and $c_{e}\left(t^{\prime}\right)$ is defined by the adjoint action of $t^{\prime}$ on $Y_{e}$, we see that $\left|c_{e}\left(t^{\prime}\right)\right|$ is bounded above and below on $\mathrm{T}_{\mathrm{U}_{0}}$. On the other hand, $c_{w^{-1}}$ is continuous and $c_{w^{-1}}(e)=0$. Hence given any $L>0$ we can find a compact open neighborhood $\mathrm{K}_{0}^{\prime}$ of $e$ such that for $k^{\prime} \in \mathrm{K}_{0}^{\prime}$ we have $\left|c_{w^{-1}}\left(k^{\prime}\right)\right| \leq q^{-L}$. In particular, we can choose $\mathrm{K}_{0}^{\prime}$ such that for all $t^{\prime} \in \mathrm{T}_{\mathrm{U}_{0}}$ and $k^{\prime} \in \mathrm{K}_{0}^{\prime}$ we have $\left|c_{e}\left(t^{\prime}\right) c_{w^{-1}}\left(k^{\prime}\right)\right|<\left|c_{w^{-1}}\left(t^{-1}\right)\right|$.

Hence, for this $\mathrm{K}_{0}^{\prime}$ we have that $\mathrm{K}_{0}^{\prime} \mathrm{T}_{\mathrm{U}_{0}} \mathrm{U}(k)$ and $S\left(w^{-1} t^{-1}\right)$ are separated by the values taken by $c_{w^{-1}}$. 
Proposition 8.6. Suppose that $w \in W$ does not support a Bessel function. Let $\mathrm{Y} \subset \mathrm{U}_{w}^{-}$be open and compact. Then for every sufficiently large open compact subset $\mathrm{U}_{0} \subset \mathrm{U}(k)$ there exists a compact open subgroup $\mathrm{K}_{0} \subset \mathrm{G}(k)$ such that if $v_{0}=v_{\mathrm{U}_{0}}$ we have the restriction of $W_{v_{0}}$ to $\mathrm{B}(k) w \mathrm{YK}_{0}$ vanishes identically.

Proof: For $\mathrm{U}_{0}$ any compact open subgroup of $\mathrm{U}(k)$ let $\mathrm{U}_{0, w}^{+}=\mathrm{U}_{0} \cap \mathrm{U}_{w}^{+}$and $\mathrm{U}_{0, w}^{-}=\mathrm{U}_{0} \cap \mathrm{U}_{w}^{-}$. By enlarging $\mathrm{U}_{0}$ if necessary, we may assume that

(i) $\mathrm{U}_{0}=\mathrm{U}_{0, w}^{+} \mathrm{U}_{0, w}^{-}$,

(ii) $Y \subset \mathrm{U}_{0, w}^{-}$

Let $\mathrm{K}_{0}=\operatorname{Stab}\left(v_{0}\right)$ and $W_{0}=W_{v_{0}}$. Then if bwyk $\in \mathrm{B}(k) w \mathrm{YK}_{0}$ with $b=u t \in$ $\mathrm{U}(k) \mathrm{T}(k)$ then $W_{0}(u t w y k)=\psi(u) W_{v_{0}}(t w) \psi(y)$.

For any $u_{0}^{+} \in \mathrm{U}_{0}^{+}$we have

$$
\psi\left(u_{0}^{+}\right) W_{0}(t w)=W_{0}\left(t w u_{0}^{+}\right)=\psi\left(A d(t) w u_{0}^{+} w^{-1}\right) W_{0}(t w) .
$$

Since $w$ does not support a Bessel function, there is a simple root $\alpha$ such that $w \alpha$ is positive but not simple. Hence for $\mathrm{U}_{0}$ sufficiently large we can find $u_{0}^{+} \in \mathrm{U}_{0, w}^{+}$lying in the root subgroup corresponding to $\alpha$ such that $\psi\left(u_{0}^{+}\right) \neq 1$ but $\psi\left(A d(t) w u_{0}^{+} w^{-1}\right) \equiv 1$ independent of $t$. Hence $W_{0}(t w) \equiv 0$.

We now consider $w \in W$ a minimal Weyl element supporting a Bessel function. Let $S_{w}=\left\{w^{\prime} \in W \mid w^{\prime} \leq w\right\}$ and let $s=s_{w}=\left|S_{w}\right|$. Enumerate the elements of $S_{w}$ so that $w_{1}^{\prime}=e, w_{s}^{\prime}=w$ and if $w_{i}^{\prime} \leq w_{j}^{\prime}$ then $i \leq j$. Recall that we have defined a canonical exhaustive sequence of open compact subgroup $\mathrm{U}_{f_{M}} \subset \mathrm{U}(k)$ in Section 1.1 indexed by positive integers $M$. In order to simplify the notation, we will now denote this subgroup by $\mathrm{U}(M)=\mathrm{U}_{f_{M}}$. Similarly, we will set $v_{M}=v_{\mathrm{U}(M)}$ and $W_{M}=W_{v_{M}}$.

Proposition 8.7. Let $w$ be a minimal Weyl element which supports a Bessel function as above. Fix a slice $S(t w)=\mathrm{U}(k)$ tw $\mathrm{U}(k)$ of the Bruhat cell $C(w)$. For every sufficiently large open compact subgroup $\mathrm{U}(M) \subset \mathrm{U}(k)$ there is a compact open subgroup $\mathrm{K}(M) \subset \mathrm{G}(k)$ such that the restriction of $W_{M}$ to the slice $S(t w)$ vanishes on $S(t w) \cap\left(\cup_{i=1}^{s-1} C\left(w_{i}^{\prime}\right) \mathrm{K}(M)\right)$, where $C\left(w_{i}^{\prime}\right)$ is the Bruhat cell $\mathrm{B}(k) w_{i}^{\prime} \mathrm{U}_{w_{i}^{\prime}}^{-}$.

Proof: We will prove this by induction on $j$. More precisely, we prove that for each $j<s$ there exists a compact open subgroup of the form $\mathrm{U}\left(M_{j}\right)$ such that for every compact open subgroup $\mathrm{U}(M) \supset \mathrm{U}\left(M_{j}\right)$ there is an 
open compact subgroup $\mathrm{K}_{j}(M) \subset \mathrm{G}(k)$ such that $W_{M}$ vanishes on $S(t w) \cap$ $\left(\cup_{i=1}^{j} C\left(w_{i}^{\prime}\right) \mathrm{K}_{j}(M)\right)$. Then the $\mathrm{U}(M)$ and $\mathrm{K}(M)$ of the theorem will be those associated to $j=s-1$.

If $j=1$ then $w_{j}^{\prime}=e$ and we may take $M_{1}=1$ and $\mathrm{U}(M)$ and $\mathrm{K}_{1}(M)$ to be those from Proposition 8.5.

We now assume the statement for $j$, that is, there exists $M_{j}$ such that for all $\mathrm{U}(M) \supset \mathrm{U}\left(M_{j}\right)$ there exists $\mathrm{K}_{j}(M)$ such that we have that $W_{M}$ vanishes on $S(t w) \cap\left(\cup_{i=1}^{j} C\left(w_{i}^{\prime}\right) \mathrm{K}_{j}(M)\right)$.

For simplicity, let us write $V_{j}(M)=\cup_{i=1}^{j} C\left(w_{i}^{\prime}\right) \mathrm{K}_{j}(M)$ and let $V_{j}=V_{j}\left(M_{j}\right)$.

Consider $C\left(w_{j+1}^{\prime}\right)$. Then $V_{j}$ is a neighborhood of $\partial C\left(w_{j+1}^{\prime}\right)=\overline{C\left(w_{j+1}^{\prime}\right)}-$ $C\left(w_{j+1}^{\prime}\right)$. Then there exists an open compact $Y_{j+1} \subset \mathrm{U}_{w_{j+1}^{\prime}}^{-}$such that

$$
\mathrm{B}(k) w_{j+1}^{\prime} Y_{j+1} \supset C\left(w_{j+1}^{\prime}\right)-V_{j} .
$$

Now take $N$ sufficiently large so that $\mathrm{U}(N)_{w_{j+1}^{\prime}}^{-} \supset Y_{j+1}$ and let $M_{j+1}=$ $\max \left(N, M_{j}\right)$.

Assume that $M^{\prime} \geq M_{j+1}$, i.e., $\mathrm{U}\left(M^{\prime}\right) \supset \mathrm{U}\left(M_{j+1}\right)$. Let

$$
V_{j}^{\prime}\left(M^{\prime}\right)=\bigcap_{u \in \mathrm{U}\left(M^{\prime}\right)} V_{j} u .
$$

Since $\left(\mathrm{U}\left(M^{\prime}\right) \cap \mathrm{K}_{j}\left(M_{j}\right)\right) \backslash \mathrm{U}\left(M^{\prime}\right)$ is finite, this is actually a finite intersection and $V_{j}^{\prime}\left(M^{\prime}\right)$ is open and still contains $\partial C\left(w_{j+1}^{\prime}\right)=\cup_{i=1}^{j} C\left(w_{j}^{\prime}\right)$. In fact, if we let

$$
\mathrm{K}_{j}^{\prime}\left(M^{\prime}\right)=\bigcap_{u \in \mathrm{U}\left(M^{\prime}\right)} u^{-1} \mathrm{~K}_{j}\left(M_{j}\right) u
$$

then

$$
V_{j}^{\prime}\left(M^{\prime}\right)=\bigcup_{i=1}^{j} C\left(w_{i}^{\prime}\right) \mathrm{K}_{j}^{\prime}\left(M^{\prime}\right)
$$

Now $C\left(w_{j+1}^{\prime}\right)-V_{j} \subset \mathrm{B}(k) w_{j+1}^{\prime} \mathrm{U}(N)_{w_{j+1}^{\prime}}^{-}$so that for all $u \in \mathrm{U}\left(M^{\prime}\right)$ we have

$$
C\left(w_{j+1}^{\prime}\right)-V_{j} u=\left(C\left(w_{j+1}^{\prime}\right)-V_{j}\right) u \subset \mathrm{B}(k) w_{j+1}^{\prime} \mathrm{U}(N)_{w_{j+1}^{\prime}}^{-} u
$$

and hence

$$
\begin{aligned}
C\left(w_{j+1}^{\prime}\right)-V_{j}^{\prime}\left(M^{\prime}\right) & \subset \bigcup_{u \in \mathrm{U}\left(M^{\prime}\right)} B(k) w_{j+1}^{\prime} \mathrm{U}(N)_{w_{j+1}^{\prime}}^{-} u \\
& =\mathrm{B}(k) w_{j+1}^{\prime} \mathrm{U}\left(M^{\prime}\right) \\
& =\mathrm{B}(k) w_{j+1}^{\prime} \mathrm{U}\left(M^{\prime}\right)_{w_{j+1}^{\prime}}^{-} .
\end{aligned}
$$


Take for $\mathrm{K}_{j+1}\left(M^{\prime}\right)$ any compact open subgroup of $\mathrm{K}_{j}^{\prime}\left(M^{\prime}\right) \cap \operatorname{Stab}\left(v_{M^{\prime}}\right)$ and let $V_{j+1}\left(M^{\prime}\right)=\cup_{i=1}^{j+1} C\left(w_{i}^{\prime}\right) \mathrm{K}_{j+1}\left(M^{\prime}\right)$. Note that

$$
V_{j+1}\left(M^{\prime}\right) \subset\left(V_{j}^{\prime}\left(M^{\prime}\right) \cup \mathrm{B}(k) w_{j+1}^{\prime} \mathrm{U}\left(M^{\prime}\right)_{w_{j-1}^{\prime}}^{-} \operatorname{Stab}\left(v_{M^{\prime}}\right)\right) .
$$

Now consider the restriction of $W_{M^{\prime}}$ to $S(t w) \cap V_{j+1}\left(M^{\prime}\right)$. Let $g \in S(t w) \cap$ $V_{j+1}\left(M^{\prime}\right)$. If $g \in V_{j}^{\prime}\left(M^{\prime}\right)$ then we write

$$
W_{M^{\prime}}(g)=\frac{1}{\operatorname{Vol}\left(\mathrm{U}\left(M^{\prime}\right)\right)} \int_{\mathrm{U}\left(M^{\prime}\right)} W_{M_{j}}(g u) \psi^{-1}(u) d u .
$$

Since $g \in V_{j}^{\prime}\left(M^{\prime}\right)$, then $g u \in V_{j}^{\prime}\left(M^{\prime}\right) u \subset V_{j}$. Since $W_{M_{j}}$ vanishes on $S(t w) \cap V_{j}$ we have $W_{M^{\prime}}(g)=0$ in this case. On the other hand, if instead $g \in S(t w) \cap$ $\mathrm{B}(k) w_{j+1}^{\prime} \mathrm{U}(M)_{w_{j+1}^{\prime}}^{-} \operatorname{Stab}\left(v_{M^{\prime}}\right)$, then

$$
W_{M^{\prime}}(g)=W_{M^{\prime}}\left(u t w_{j+1}^{\prime} u_{j+1}^{-} k\right)=\psi(u) W_{0}\left(t w_{j+1}^{\prime}\right) \psi\left(u_{j+1}^{-}\right) .
$$

But since $w_{j+1}^{\prime}$ does not support a Bessel function, we see that $W_{M^{\prime}}\left(t w_{j+1}^{\prime}\right)=0$ as in the proof of Proposition 8.6.

Therefore $W_{M^{\prime}}$ vanishes on $S(t w) \cap V_{j+1}\left(M^{\prime}\right)$. This completes the induction and thereby the proof of the proposition.

This proposition then leads to the existence of $J_{\pi, w}$ for $w$ a minimal Weyl element that supports a Bessel function. To see this, consider

$$
J_{\pi, w}(a)=\int_{\mathrm{U}_{w}^{-}} W_{v}(a w u) \psi^{-1}(u) d u
$$

for $a \in \mathrm{A}_{w}$ and $v \in V_{\pi}$ with $W_{v}(e)=1$. Note that for any $M$ we have by an elementary change of variables that

$$
\int_{\mathrm{U}_{w}^{-}} W_{v}(a w u) \psi^{-1}(u) d u=\int_{\mathrm{U}_{w}^{-}} W_{v_{M}}(a w u) \psi^{-1}(u) d u
$$

in the notation of Proposition 8.7. Fixing $a \in \mathrm{A}_{w}$, by Proposition 8.7 there exists an $M^{\prime}$ such that $W_{v_{M^{\prime}}}$ vanishes on $S(a w) \cap\left(\cup_{i=1}^{s-1} \mathrm{~B}(k) C\left(w_{j}^{\prime}\right) \mathrm{K}\left(M^{\prime}\right)\right.$, that is, $W_{v_{M^{\prime}}}(a w u)$, as a function of $u \in \mathrm{U}_{w}^{-}$, is compactly supported. Hence this integral converges and $J_{\pi, w}(a)$ exists.

Theorem 8.8. If $\pi$ is a generic representation of $\mathrm{G}(k)$ and $w$ is a minimal Weyl element that supports a Bessel function, then the associated (full) Bessel function $J_{\pi, w}(a)$ exists as a function on $\mathrm{A}_{w}$. 


\section{References}

[1] Z. Averbuch, Bessel functions of algebraic groups' representations over a local field. Theses, Tel-Aviv University, 1992.

[2] A. Borel, Linear Algebraic Groups. Graduate Texts in Mathematics 126. Springer-Verlag, New York, 1991.

[3] A. Borel and J. Tits, Groupes réductifs. Publ. Math. IHES 27 (1965), 55-150.

[4] F. Bruhat and J. Tits, Groupes réductifs sur un corps local, Chapitre I. Publ. Math. IHES 41 (1972), 5-252.

[5] F. Bruhat and J. Tits, Groupes réductifs sur un corps local, Chapitre II. Publ. Math. IHES 60 (1984), 5-184.

[6] J.W. Cogdell, H.H. Kim, I.I. Piatetski-Shapiro, and F. Shahidi, On lifting from classical groups to $G L_{N}$. Publ. Math. IHES 79 (2001), 5-30.

[7] J.W. Cogdell, H.H. Kim, I.I. Piatetski-Shapiro, and F. Shahidi, Functoriality for the classical groups, Publ. Math. IHES 99 (2004), 163-233.

[8] J.W. Cogdell and I.I. Piatetski-Shapiro, Stability of gamma factors for $\mathrm{SO}(2 n+$ 1), manuscripta math. 95 (1998), 437-461.

[9] J.W. Cogdell, I.I. Piatetski-Shapiro, and F. Shahidi, Stability of $\gamma$-factors for quasi-split groups. In preparation.

[10] I.M. Gelfand and M.I. Graev, The construction of irreducible representations of simple algebraic groups over a finite field. Dokl. Akad. Nauk USSR 147 (1962), 529-532.

[11] S.I. Gelfand, Representations of the general linear group over a finite field. Lie Groups and Their Representations (I.M. Gelfand, Ed.), John Wiley and Sons, New York, 1975, 119-132.

[12] J.E. Humphreys, Reflection Groups and Coxeter Groups. Cambridge Studies in Advanced mathematics 29. Cambridge University Press, Cambridge, 1990.

[13] I. Satake, Classification Theory of Semi-simple Algebraic Groups. Lecture Notes in Pure and Applied Mathematics 3. Marcel Dekker, New York, 1971.

[14] F. Shahidi, Local coefficients as Artin factors for real groups. Duke Math. J. 52 (1985), 973-1007.

[15] F. Shahidi, A proof of Langlands' conjecture on Plancherel measures; Complementary series for p-adic groups. Annals of Math. 132 (1990), 273-330.

[16] F. Shahidi, Local coefficients as Mellin transforms of Bessel functions: Towards a general stability. IMRN 2002, No. 39, (2002), 2075-2119.

[17] R. Steinberg, Lectures on Chevalley Groups. Mimeographed notes, Yale University, 1967. 
128 J.W. Cogdell, I.I. Piatetski-Shapiro and F. Shahidi

J. W. Cogdell

Department of Mathematics

Oklahoma State University

Stillwater, OK 74078, USA

Current address:

Department of Mathematics

Ohio State University

Columbus, OH 43210, USA

cogdell@math.ohio-state.edu

I. I. Piatetski-Shapiro

Department of Mathematics

Yale University

New Haven, CT 06520, USA

and

School of Mathematics

Tel Aviv University

Tel Aviv 69978, Israel

ilya@math.yale.edu

\section{F. Shahidi}

Department of Mathematics

Purdue University

West Lafayette, IN 47907, USA

shahidi@math.purdue.edu 